F. Catanese, M. Franciosi, K. Hulek and M. Reid

Nagoya Math. J.

Vol. 154 (1999), 185-220

\title{
EMBEDDINGS OF CURVES AND SURFACES
}

\author{
FABRIZIO CATANESE, MARCO FRANCIOSI, \\ KLAUS HULEK AND MILES REID
}

\begin{abstract}
We prove a general embedding theorem for Cohen-Macaulay curves (possibly nonreduced), and deduce a cheap proof of the standard results on pluricanonical embeddings of surfaces, assuming vanishing $H^{1}\left(2 K_{X}\right)=0$.
\end{abstract}

\section{$\S 1$. Introduction}

Let $C$ be a curve over an algebraically closed field $k$ of characteristic $p \geq 0$, and $H$ a Cartier divisor on $C$. We assume that $C$ is projective and Cohen-Macaulay (but possibly reducible or nonreduced). Write $H C=$ $\operatorname{deg} \mathcal{O}_{C}(H)$ for the degree of $H, p_{a} C=1-\chi\left(\mathcal{O}_{C}\right)$ for the arithmetic genus of $C$, and $\omega_{C}$ for the dualising sheaf (see [Ha], Chap. III, $\S 7$ ).

Our first result is the following. (A cluster $Z$ of degree $\operatorname{deg} Z=r$ is simply a 0-dimensional subscheme with length $\mathcal{O}_{Z}=\operatorname{dim}_{k} \mathcal{O}_{Z}=r$; a curve $B$ is generically Gorenstein if, outside a finite set, $\omega_{B}$ is locally isomorphic to $\mathcal{O}_{B}$. The remaining definitions and notation are explained below.)

Theorem 1.1. (Curve embedding theorem) $H$ is very ample on $C$ if for every generically Gorenstein subcurve $B \subset C$, either

1. $H B \geq 2 p_{a} B+1$, or

2. $H B \geq 2 p_{a} B$, and there does not exist a cluster $Z \subset B$ of degree 2 such that $\mathcal{I}_{Z} \mathcal{O}_{B}(H) \cong \omega_{B}$.

More generally, suppose that $Z \subset C$ is a cluster (of any degree) such that the restriction

$$
H^{0}\left(C, \mathcal{O}_{C}(H)\right) \longrightarrow \mathcal{O}_{Z}(H)=\mathcal{O}_{C}(H) \otimes \mathcal{O}_{Z}
$$

Received May 7, 1997.

Research carried out under the EU HCM project AGE (Algebraic Geometry in Europe), contract number ERBCHRXCT 940557. The final version was written while the first author was "Professore distaccato" at the Accademia dei Lincei. 
is not onto. Then there exists a generically Gorenstein subcurve $B$ of $C$ and an inclusion $\varphi: \mathcal{I}_{Z} \mathcal{O}_{B}(H) \hookrightarrow \omega_{B}$ not induced by a map $\mathcal{O}_{B}(H) \rightarrow \omega_{B}$. In particular, (1) is onto if

$$
H B>2 p_{a} B-2+\operatorname{deg}(Z \cap B)
$$

for every generically Gorenstein subcurve $B \subset C$.

Theorem 1.1 is well known for nonsingular curves $C$. Although particular cases were proved in $[\mathrm{Ca} 1],[\mathrm{Ba} 2],[\mathrm{C}-\mathrm{F}],[\mathrm{C}-\mathrm{H}]$, it was clear that the result was more general. In discussion after a lecture on the Gorenstein case by the first author at the May 1994 Lisboa AGE meeting, the fourth author pointed out the above result, where $C$ is only assumed to be a pure 1-dimensional scheme. For divisors on a nonsingular surface, Mendes Lopes [ML] has obtained results analogous to Theorem 1.1 and to Theorem 3.6. We apply these ideas to the canonical map of a Gorenstein curve in $\S 3$.

The proof of Theorem 1.1 is based on two ideas from Serre and Grothendieck duality:

(a) we use Serre duality in its "raw" form

$$
H^{1}(C, \mathcal{F}) \underline{\mathrm{d}} \operatorname{Hom}\left(\mathcal{F}, \omega_{C}\right) \quad \text { for } \mathcal{F} \text { a coherent sheaf, }
$$

where $\underline{d}$ denotes duality of vector spaces.

(b) If $\mathcal{O}_{C}$ has nilpotents, a nonzero map $\varphi: \mathcal{F} \rightarrow \omega_{C}$ is not necessarily generically onto; however (because we are Hom'ming into $\omega_{C}$ ), duality gives an automatic factorisation of $\varphi$ of the form

$$
\mathcal{F} \longrightarrow \mathcal{F}_{\mid B} \longrightarrow \omega_{B} \longrightarrow \omega_{C},
$$

via a purely 1-dimensional subscheme $B \subset C$, where $\mathcal{F}_{\mid B} \rightarrow \omega_{B}$ is generically onto. See Lemma 2.4 for details.

Since our main result might otherwise seem somewhat abstract and useless, we motivate it by giving a short proof in $\S 4$, following the methods of $[\mathrm{C}-\mathrm{F}]$, of the following result essentially due to Bombieri (when char $k=0$ ) and to Ekedahl and Shepherd-Barron in general. Recall that a canonical surface (or canonical model of a surface of general type) is a surface with at worst Du Val singularities and $K_{X}$ ample. The remaining notation and definitions are explained below. 
Theorem 1.2. (Canonical embeddings of surfaces) $X$ is a canonical surface. Assume that $H^{1}\left(2 K_{X}\right)=0$. Then $m K_{X}$ is very ample if $m \geq 5$, or if $m=4$ and $K_{X}^{2} \geq 2$, or if $m=3, p_{g} \geq 2$ and $K_{X}^{2} \geq 3$.

Here $H^{1}\left(2 K_{X}\right)=0$ follows at once in characteristic 0 from Kodaira vanishing or Mumford's vanishing theorem. One can also get around the assumption $H^{1}\left(2 K_{X}\right)=0$ in characteristic $p>0$ (see [Ek] or [S-B]). In fact Ekedahl's analysis (see [Ek, Theorem II.1.7]) shows that $H^{1}\left(2 K_{S}\right) \neq 0$ is only possible in a very special case, when $p=2, \chi\left(\mathcal{O}_{S}\right)=1$ and $S$ is (birationally) an inseparable double cover of a K3 surface or a rational surface. (The condition $p_{g} \geq 2$ when $m=3$ is needed for the simple minded proof of $\S 4$, but not for the validity of the theorem.)

In $\S 5$ and $\S 6$ we apply these ideas to prove the following theorems on tricanonical and bicanonical linear systems of a surface of general type.

THEOREM 1.3. (Tricanonical embeddings) Suppose that $X$ is a canonical surface with $K_{X}^{2} \geq 3$. Then $3 K_{X}$ is very ample if either

(a) $q^{\prime}:=h^{1}\left(\mathcal{O}_{X}\right)=0 ;$ or

(b) $\chi\left(\mathcal{O}_{X}\right) \geq 1, \operatorname{dim} \operatorname{Pic}^{0} X>0$ and $H^{1}\left(2 K_{X}-L\right)=0$ for all $L \in \operatorname{Pic}^{0} X$.

Note that (a) or (b) cover all cases with char $k=0$. Thus the cases not covered by our argument are in char $k=p>0$, with either $p_{g}<q^{\prime}$ or $\operatorname{dim} \operatorname{Pic}^{0} X=0$.

Theorem 1.3 in characteristic 0 is a result of Reider [Rei], but see also [Ca2]. Without the condition $K_{X}^{2} \geq 3$, the double plane with branch curve of degree 8 (that is, $X_{8} \subset \mathbb{P}(1,1,1,4)$ ) is a counterexample. It follows from a result of Ekedahl ([Ek, Theorem II.1.7]) that if $\chi\left(\mathcal{O}_{X}\right) \geq 1$ then $H^{1}\left(2 K_{X}-L\right)=0$ for all $L \neq 0$. The remaining assumption in Theorem 1.3 is that $H^{1}\left(2 K_{X}\right)=0$, and this can also be got around, as shown by Shepherd-Barron [S-B].

THEOREM 1.4. (Bicanonical embeddings) We now assume that $q^{\prime}=0$ and $p_{g} \geq 4$.

(a) $2 K_{X}$ is very ample if every $C \in\left|K_{X}\right|$ is numerically 3-connected (in the sense of Definition 3.1, see also Lemma 4.2). More precisely, $\left|2 K_{X}\right|$ separates a cluster $Z$ of degree 2 provided that every curve $C \in\left|K_{X}\right|$ through $Z$ is 3-connected. 
(b) Assume in addition that $K_{X}^{2} \geq 10$, and let $Z$ be a cluster of degree 2 contained in $X$. Then $Z$ is contracted by $\left|2 K_{X}\right|$ if and only if $Z$ is contained in a curve $B \subset X$ with

$$
K_{X} B=p_{a} B=1 \text { or } 2
$$

( a Francia curve, compare Definition 6.1), and $\mathcal{I}_{Z} \mathcal{O}_{B}\left(2 K_{X}\right) \cong \omega_{B}$.

(c) In particular, $\left|2 K_{X}\right|$ defines a birational morphism unless $X$ has a pencil of curves of genus 2.

Remarks 1.5. (1) See Definition 6.1 for more on Francia curves. A cluster $Z$ of degree 2 is automatically contracted by $\left|2 K_{X}\right|$ if it is contained in a curve $C \subset X$ for which $\mathcal{I}_{Z} \mathcal{O}_{C}\left(2 K_{X}\right) \cong \omega_{C}$ (for a nonsingular curve, this reads $2 K_{X \mid C}=K_{C}+P+Q$ ). Thus (b) says in particular that if this happens for some $C$ then it also happens for a Francia curve.

(2) The assumptions $q^{\prime}=0$ and $p_{g} \geq 4$ are needed for the simple minded "restriction method" of this paper, but we conjecture that (b) holds without them (at least in characteristic zero, or assuming $q^{\prime}=0$ ); the case $Z=\{x, y\}$ with $x \neq y$ (that is, "separating points") follows in characteristic zero by Reider's method. We believe that the conjecture can be proved quite generally by a different argument based on Ramanujam-Francia vanishing, or by Reider's method applied to reflexive sheaves on $X$. Stay tuned!

(3) In characteristic 0, Theorem 1.4 (without the assumption $q^{\prime}=0$ ) is due essentially to Francia (unpublished, but see $[\mathrm{Fr} 1]-[\mathrm{Fr} 2]$ ) and Reider [Rei]. Theorem 1.4, (a) is a consequence of Theorem 3.6 on canonical embeddings of curves and the generalisation of hyperelliptic curves. The results in Theorem 1.4 are only a modest novelty, in that there is no restriction on the characteristic of the ground field (see [S-B, Theorems 25, 26 and 27] for char $k \geq 11$ ).

Further results on the bicanonical map $\varphi_{2 K}$ for smaller values of $p_{g}$, $K_{X}^{2}$ (in characteristic 0 ) require a more intricate analysis, and we refer to recent or forthcoming articles $([\mathrm{C}-\mathrm{F}-\mathrm{M}],[\mathrm{C}-\mathrm{C}-\mathrm{M}])$. Other applications of our methods can be found in $[\mathrm{F}]$.

Acknowledgements. It is a pleasure to thank Ingrid Bauer for interesting discussions on linear systems on surfaces, out of which this paper originated. 


\section{Conventions}

This paper deals systematically with reducible and nonreduced curves and their subschemes $B \subset C$. A coherent sheaf $\mathcal{F}$ on a curve $C$ is torsion free if there are no sections $s \in \mathcal{F}$ supported at points; on a 1-dimensional scheme, this is obviously equivalent to $\mathcal{F}$ Cohen-Macaulay. We say that $C$ is purely 1-dimensional or Cohen-Macaulay if $\mathcal{O}_{C}$ is torsion free.

A map $\varphi: \mathcal{F} \rightarrow \mathcal{G}$ between coherent sheaves on $B$ is generically injective if it is injective at every generic point of $B$; if $\mathcal{F}$ is torsion free then $\varphi$ is automatically an inclusion $\mathcal{F} \hookrightarrow \mathcal{G}$. If we know that the generic stalks of $\mathcal{F}$ and $\mathcal{G}$ have the same length at every generic point of $C$ then a generically injective map $\varphi: \mathcal{F} \rightarrow \mathcal{G}$ is an isomorphism at each generic point, and therefore $\operatorname{ker} \varphi$ and $\operatorname{coker} \varphi$ have finite length. Indeed, they are both coherent sheaves supported at a finite set, and by the Nullstellensatz, each stalk is killed by a power of the maximal ideal. This applies, for example, to the $\operatorname{map} \varphi: \mathcal{I}_{Z} \mathcal{O}_{B}(H) \hookrightarrow \omega_{B}$ of Theorem 1.1, see Lemma 2.3 below.

A scheme $B$ is Gorenstein in codimension 0 or generically Gorenstein if $\omega_{B}$ is locally isomorphic to $\mathcal{O}_{B}$ at every generic point of $B$.

A cluster of degree $r$ is a 0 -dimensional subscheme $Z \subset X$ supported at finitely many points, with ideal sheaf $\mathcal{I}_{Z}$, structure sheaf $\mathcal{O}_{Z}=\mathcal{O}_{X} / \mathcal{I}_{Z}$, and having $\operatorname{deg} Z=h^{0}\left(\mathcal{O}_{Z}\right)=\operatorname{length} \mathcal{O}_{Z}=r$. We sometimes write $Z=(x, y)$ for a cluster of degree 2 , where $x, y$ are either 2 distinct points of $X$, or a point $x$ plus a tangent vector $y$ at $x$. We say that a linear system $|H|$ on $X$ separates $Z$ (or separates $x$ and $y$ ) if $H^{0}\left(X, \mathcal{O}_{X}(H)\right) \rightarrow \mathcal{O}_{Z}(H)$ is onto, or contracts $Z$ if $Z$ does not meet the base locus Bs $|H|$, and $\operatorname{rank}\left\{H^{0}\left(X, \mathcal{O}_{X}(H)\right) \rightarrow \mathcal{O}_{Z}(H)\right\}=1$.

\section{Notation}

$X \quad$ A projective scheme over an algebraically closed field $k$; however, our main conclusions work over an arbitrary field, except insofar as they involve choosing a point or a cluster of given degree (for example, to see that a genus 0 curve is $\cong \mathbb{P}^{1}$ in Theorem 3.3 , or a genus 1 curve has a $g_{2}^{1}$ in Theorem 3.6).

$\omega_{X} \quad$ Dualising sheaf of $X$ (see [Ha, Chap. III, $\left.\S 7\right]$ ).

$|H| \quad$ Linear system defined by a Cartier divisor $H$ on $X$.

$C \quad$ A curve, that is, a projective scheme over $k$ which is purely 1-dimensional, in the sense that $\mathcal{O}_{C}$ is Cohen-Macaulay (torsion free).

$p_{a} C$ The arithmetic genus of $C, p_{a} C=1-\chi\left(\mathcal{O}_{C}\right)$. 
$K_{C} \quad$ A canonical divisor of a Gorenstein curve $C$, that is, a Cartier divisor such that $\mathcal{O}_{C}\left(K_{C}\right) \cong \omega_{C}$ (only defined if $C$ is Gorenstein).

$\operatorname{deg} \mathcal{L}$ The degree of a torsion free sheaf of rank 1 on $C$; it can be defined by

$$
\operatorname{deg} \mathcal{L}=\chi(\mathcal{L})-\chi\left(\mathcal{O}_{C}\right)
$$

If $H$ is a Cartier divisor on $C$, we set $H C=\operatorname{deg} \mathcal{O}_{C}(H)$.

$S \quad$ A nonsingular projective surface.

$D D^{\prime}$ Intersection number of divisors $D, D^{\prime}$ on a nonsingular projective surface.

$K_{S} \quad$ A canonical divisor on $S$.

$K_{X}^{2}$ If $X$ is a Gorenstein surface, $K_{X}$ is a Cartier divisor with $\omega_{X}=$ $\mathcal{O}_{X}\left(K_{X}\right)$, and $K_{X}^{2}$ is the selfintersection number of the Cartier divisor $K_{X}$. If $X$ has only Du Val singularities and $\pi: S \rightarrow X$ is the minimal nonsingular model then $K_{S}=\pi^{*} K_{X}$ and $K_{X}^{2}=K_{S}^{2}$.

$p_{g}, q^{\prime}$ The geometric genus $p_{g}=h^{0}\left(S, K_{S}\right)=h^{0}\left(X, K_{X}\right)$ of $S$ or $X$ (respectively the irregularity $\left.q^{\prime}=h^{1}\left(S, \mathcal{O}_{S}\right)=h^{1}\left(X, \mathcal{O}_{X}\right)\right)$.

$P_{n} \quad$ The $n$th plurigenus $P_{n}=h^{0}\left(S, n K_{S}\right)$ of $S$.

\section{$\S 2$. Embedding curves}

We start with a useful remark.

Remark 2.1. $\quad$ Let $H$ be a Cartier divisor on a scheme $X$. Then $H$ is very ample if and only if the restriction map

$$
H^{0}\left(\mathcal{O}_{X}(H)\right) \longrightarrow \mathcal{O}_{Z}(H)
$$

is onto for every cluster $Z \subset X$ of degree $\leq 2$.

Proof. By the standard embedding criterion of [Ha, Chap. II, Prop.7.3], we have to prove that $(2)$ is onto for all the ideals $\mathcal{I}_{Z}=m_{x}$ or $m_{x} m_{y}$ with $x, y \in X$. For $x \neq y$, we are done.

By assumption $H^{0}\left(\mathcal{O}_{C}(H)\right) \rightarrow \mathcal{O}_{C} / m_{x}$ is onto for every $x \in X$. Now if the image of $H^{0}\left(m_{x} \mathcal{O}_{C}(H)\right) \rightarrow m_{x} / m_{x}^{2}$ is contained in a hyperplane $V \subset$ $m_{x} / m_{x}^{2}$, then the inverse image of $V$ in $\mathcal{O}_{C, x}$ generates an ideal $\mathcal{I} \subset \mathcal{O}_{X, x}$ defining a cluster $Z$ of degree 2 supported at $x$ such that $H^{0}\left(\mathcal{O}_{C}(H)\right) \rightarrow \mathcal{O}_{Z}$ is not onto.

Q.E.D. 
Remark 2.2. The chain of reasoning we use below is that, by Remark 2.1 and cohomology, $H$ is very ample if and only if $H^{1}\left(\mathcal{I}_{Z} \mathcal{O}_{X}(H)\right) \rightarrow$ $H^{1}\left(\mathcal{O}_{X}(H)\right)$ is injective for each cluster $Z$ of degree 2 , or dually (if $X=C$ is a curve), $\operatorname{Hom}\left(\mathcal{O}_{C}(H), \omega_{C}\right) \rightarrow \operatorname{Hom}\left(\mathcal{I}_{Z}(H), \omega_{C}\right)$ is onto.

LEMma 2.3. Let $C$ be a curve. Assume that there is a Cartier divisor $H$ on $C$ and a cluster $Z \subset C$ for which the sheaf $\mathcal{L}=\mathcal{I}_{Z} \mathcal{O}_{C}(H)$ has an inclusion $\mathcal{L} \hookrightarrow \omega_{C}$. Then $C$ is generically Gorenstein.

Proof. By assumption, $\mathcal{L} \cong \mathcal{O}_{C}$ at every generic point of $C$. We must prove that an inclusion $\mathcal{L} \hookrightarrow \omega_{C}$ maps onto every generic stalk $\omega_{C, \eta}$, or equivalently, that the cokernel $\mathcal{N}=\omega_{C} / \mathcal{L}$ has finite length. We give two slightly different proofs, one based on RR, and one using properties of dualising modules.

Let $\mathcal{O}_{C}(1)$ be an ample line bundle on $C$. Then by Serre vanishing (see [Se1, $\mathrm{n}^{\circ}$ 66, Theorem 2] or [Ha, Chap. III, Theorem 5.2]), for $n \gg 0$, the exact sequence

$$
0 \longrightarrow \mathcal{L}(n) \longrightarrow \omega_{C}(n) \longrightarrow \mathcal{N}(n) \longrightarrow 0
$$

is exact on global sections, and all the $H^{1}$ vanish. Now by RR and duality,

$$
h^{0}\left(\omega_{C}(n)\right)=h^{1}\left(\mathcal{O}_{C}(-n)\right)=-\chi\left(\mathcal{O}_{C}\right)+n \operatorname{deg} \mathcal{O}_{C}(1) \quad \text { for } n \gg 0 .
$$

On the other hand, RR also gives $h^{0}(\mathcal{L}(n))=\chi\left(\mathcal{O}_{C}\right)+\operatorname{deg} \mathcal{L}+n \operatorname{deg} \mathcal{O}_{C}(1)$ for $n \gg 0$, since $\mathcal{L} \cong \mathcal{O}_{C}$ at every generic point. Thus

$$
h^{0}(\mathcal{N}(n))=-2 \chi\left(\mathcal{O}_{C}\right)+\operatorname{deg} \mathcal{L} \text { for all } n \gg 0
$$

and therefore $\mathcal{N}$ has finite length.

The alternative proof of the lemma uses the "well-known fact" (see below) that the generic stalk $\omega_{C, \eta}$ of the dualising sheaf at a generic point $\eta \in C$ is a dualising module for the Artinian local ring $\mathcal{O}_{C, \eta}$, so that they have the same length, and therefore an inclusion $\mathcal{L} \hookrightarrow \omega_{C}$ is generically an isomorphism. The above proof in effect deduces length $\omega_{C, \eta}=\operatorname{length} \mathcal{O}_{C, \eta}$ from RR together with Serre duality, the defining property of $\omega_{C}$.

Proof of the "well-known fact". This is a proof by incomprehensible reference. First, if $\eta \in X$ is a generic point of a scheme, more-or-less by definition, a dualising module of the Artinian $\operatorname{ring} \mathcal{O}_{X, \eta}$ is an injective hull of the 
residue field $\mathcal{O}_{X, \eta} / m_{X, \eta}=k(\eta)$ (see [Gr-Ha, Proposition 4.10]); in simpleminded terms, $\mathcal{O}_{X, \eta}$ clearly contains a field $K_{0}$ such that $K_{0} \subset k(\eta)$ is a finite field extension, and the vector space dual $\operatorname{Hom}_{K_{0}}\left(\mathcal{O}_{X, \eta}, K_{0}\right)$ is a dualising module. Next, if $\eta \in X$ is a generic point of a subscheme $X \subset \mathbb{P}=\mathbb{P}^{N}$ of pure codimension $r$, then by [Ha, Chap. III, Prop. 7.5], the dualising sheaf of $X$ is $\omega_{X}=\mathcal{E} x t_{\mathcal{O}_{\mathbb{P}}}^{r}\left(\mathcal{O}_{X}, \omega_{\mathbb{P}}\right)$. On the other hand, the local ring $\mathcal{O}_{\mathbb{P}, \eta}$ of projective space along $\eta$ is an $r$-dimensional regular local ring, and therefore Gorenstein, so that by [Gr-Ha, Prop. 4.13], $\operatorname{Ext}_{\mathcal{O}_{\mathbb{P}, \eta}}^{r}\left(\mathcal{O}_{X, \eta}, \omega_{\mathbb{P}, \eta}\right)$ is a dualising module of $\mathcal{O}_{X, \eta}$ (an injective hull of the residue field $\mathcal{O}_{X, \eta} / m_{X, \eta}=$ $k(\eta))$.

Q.E.D.

LEMma 2.4. (Automatic adjunction) Let $\mathcal{F}$ be a coherent sheaf on $C$, and $\varphi: \mathcal{F} \rightarrow \omega_{C}$ a map of $\mathcal{O}_{C}$-modules. Set $\mathcal{J}=$ Ann $\varphi \subset \mathcal{O}_{C}$, and write $B \subset C$ for the subscheme defined by $\mathcal{J}$. Then $B$ is Cohen-Macaulay and $\varphi$ has a canonical factorisation of the form

$$
\mathcal{F} \longrightarrow \mathcal{F}_{\mid B} \longrightarrow \omega_{B}=\mathcal{H o m}_{\mathcal{O}_{C}}\left(\mathcal{O}_{B}, \omega_{C}\right) \subset \omega_{C}
$$

where $\mathcal{F}_{\mid B} \rightarrow \omega_{B}$ is generically onto.

Proof. First, $\omega_{C}$ is torsion free, because $\operatorname{Hom}\left(\mathcal{G}, \omega_{C}\right)=0$ for any sheaf $\mathcal{G}$ with 0 dimensional support, hence $\mathcal{J}=\operatorname{Ann} \varphi$ has no embedded primes, and $\mathcal{O}_{B}=\mathcal{O}_{C} / \mathcal{J}$ is Cohen-Macaulay. By construction of $\mathcal{J}$, the image of $\varphi$ is contained in the submodule

$$
\left\{s \in \omega_{C} \mid \mathcal{J} s=0\right\} \subset \omega_{C}
$$

But this clearly coincides with $\mathcal{H o m}\left(\mathcal{O}_{B}, \omega_{C}\right)$. Now the inclusion morphism $B \hookrightarrow C$ is finite, and $\omega_{B}=\mathcal{H o m}_{\mathcal{O}_{C}}\left(\mathcal{O}_{B}, \omega_{C}\right)$ is just the adjunction formula for a finite morphism (see, for example, [Ha, Chap. III, §7, Ex. 7.2], or [Re, Prop. 2.11]).

The factorisation (3) goes like this: $\varphi$ is killed by $\mathcal{J}$, so it factors via the quotient module $\mathcal{F} / \mathcal{J} \mathcal{F}=\mathcal{F}_{\mid B}$. As just observed, it maps into $\omega_{B} \subset \omega_{C}$. Finally, it maps onto every generic stalk of $\omega_{B}$, again by definition of $\mathcal{J}$ : a submodule of the sum of generic stalks $\bigoplus \omega_{B, \eta}$ is the dual to the generic stalk $\bigoplus \mathcal{O}_{B^{\prime}, \eta}$ of a purely 1-dimensional subscheme $B^{\prime} \subset B$, and $\varphi$ is not killed by the corresponding ideal sheaf $\mathcal{J}^{\prime}$.

Q.E.D.

Remark 2.5. We define $B$ to be the scheme theoretic support of $\varphi$. Note that if $C=\sum n_{i} \Gamma_{i}$ is a Weil divisor on a normal surface and $\mathcal{F}$ a line bundle, 
the curve $B \subset C$ defines a splitting $C=A+B$ where $A$ is the divisor of zeros of $\varphi$ : at the generic point of $\Gamma_{i}$, the map $\varphi$ then looks like $y_{i}^{a_{i}}$, where $y_{i}$ is the local equation of $\Gamma_{i}$, and $A=\sum a_{i} \Gamma_{i}$. In the general case however, $A$ does not make sense.

Proof of Theorem 1.1. Let $H$ be a Cartier divisor, and $\mathcal{I}$ the ideal sheaf of a cluster for which $H^{1}\left(\mathcal{I O}_{C}(H)\right) \neq 0$. Then $\operatorname{Hom}\left(\mathcal{I} \mathcal{O}_{C}(H), \omega_{C}\right) \neq 0$ by Serre duality. First pick any nonzero map $\varphi: \mathcal{I O}_{C}(H) \rightarrow \omega_{C}$. By Lemma 2.4, $\varphi$ comes from an inclusion $\mathcal{I O}_{B}(H) \hookrightarrow \omega_{B}$ for a subscheme $B \subset C$, and $B$ is generically Gorenstein by Lemma 2.3.

Finally, if $H^{0}\left(\mathcal{O}_{C}(H)\right) \rightarrow \mathcal{O}_{Z}(H)$ is not onto, then the next arrow in the cohomology sequence

$$
H^{1}\left(\mathcal{I O}_{C}(H)\right) \longrightarrow H^{1}\left(\mathcal{O}_{C}(H)\right)
$$

is not injective, and dually, the restriction map

$$
\operatorname{Hom}\left(\mathcal{O}_{C}(H), \omega_{C}\right) \longrightarrow \operatorname{Hom}\left(\mathcal{I} \mathcal{O}_{C}(H), \omega_{C}\right)
$$

is not onto. Thus we can pick $\varphi: \mathcal{I O}_{C}(H) \rightarrow \omega_{C}$ which is not the restriction of a map $\mathcal{O}_{C}(H) \rightarrow \omega_{C}$. Then also the map $\mathcal{I O}_{B}(H) \hookrightarrow \omega_{B}$ given by Lemma 2.4 is not the restriction of a $\operatorname{map} \mathcal{O}_{B}(H) \hookrightarrow \omega_{B}$.

For the final part, an inclusion $\mathcal{I O}_{B}(H) \hookrightarrow \omega_{B}$ has cokernel of finite length, so that $\chi\left(\mathcal{I} \mathcal{O}_{B}(H)\right) \leq \chi\left(\omega_{B}\right)$. Plugging in the definition of degree gives

$$
1-p_{a} B+H B-\operatorname{deg}(Z \cap B) \leq p_{a} B-1
$$

that is,

$$
H B \leq 2 p_{a} B-2+\operatorname{deg}(Z \cap B) .
$$

Thus, assuming the inequality (2) of Theorem 1.1, no such inclusion $\mathcal{I O}_{B}(H) \hookrightarrow \omega_{B}$ can exist, so that $H^{0}\left(\mathcal{O}_{C}(H)\right) \rightarrow \mathcal{O}_{Z}(H)$ is onto. Q.E.D.

\section{$\S 3$. The canonical map of a Gorenstein curve}

We now discuss the canonical map $\varphi_{K_{C}}$ of a Gorenstein curve, writing $K_{C}$ for a canonical divisor of $C$, that is, a Cartier divisor for which $\omega_{C} \cong$ $\mathcal{O}_{C}\left(K_{C}\right)$. Our approach is motivated in part by the examples and results in the reduced case treated in [Ca1]. 
Definition 3.1. A Gorenstein curve $C$ is numerically m-connected if

$$
\operatorname{deg} \mathcal{O}_{B}\left(K_{C}\right)-\operatorname{deg} \omega_{B}=\operatorname{deg}\left(\omega_{C} \otimes \mathcal{O}_{B}\right)-\left(2 p_{a} B-2\right) \geq m
$$

for every generically Gorenstein strict subcurve $B \subset C$. (We say that a curve $C$ over any field $k$ is numerically $m$-connected if $C \otimes \bar{k}$ is.)

Remark 3.2. Note that for divisors on a nonsingular surface,

$$
\operatorname{deg} \mathcal{O}_{B}\left(K_{C}\right)-\operatorname{deg} \omega_{B}=\left(K_{S}+C\right) B-\left(K_{S}+B\right) B=(C-B) B
$$

In this context, Franchetta and Ramanujam define numerically connected in terms of the intersection numbers $A B=(C-B) B$ for all effective decompositions $C=A+B$. The point of our definition is to use the numbers $\operatorname{deg} \mathcal{O}_{B}\left(K_{C}\right)-\operatorname{deg} \omega_{B}$ in the more general case as a substitute for $(C-B) B$. In effect, we think of the adjunction formula as defining the "degree" of the "normal bundle" to $B$ in $C$, in terms of the difference between $K_{C \mid B}$ and $\omega_{B}$.

\section{THEOREM 3.3. Let $C$ be a Gorenstein curve.}

(a) If $C$ is numerically 1-connected then $H^{0}\left(\mathcal{O}_{C}\right)=k$ (the constant functions).

(b) If $C$ is numerically 2-connected then either $\left|K_{C}\right|$ is free or $C \cong \mathbb{P}^{1}$. In particular, in this case $p_{a} C=0$ implies $C \cong \mathbb{P}^{1}$.

Proof of (a). First, if $f \in H^{0}\left(\mathcal{O}_{C}\right)$ is a nonzero section vanishing along some reduced component of $C$, then applying Lemma 2.4 to the multiplication map $\mu_{f}: \mathcal{O}_{C}\left(K_{C}\right) \rightarrow \omega_{C}$ gives an inclusion $\mathcal{O}_{B}\left(K_{C}\right) \hookrightarrow \omega_{B}$, which numerically 1-connected forbids (because $\operatorname{deg} \mathcal{O}_{B}\left(K_{C}\right)>\operatorname{deg} \omega_{B}$ ). Now if $h^{0}\left(\mathcal{O}_{C}\right)>1$, there exists a nonzero section $f \in H^{0}\left(\mathcal{O}_{C}\right)$ vanishing at any given point $x \in C$. An inclusion $\mathcal{O}_{C} \hookrightarrow m_{x}$ contradicts at once $0=\operatorname{deg} \mathcal{O}_{C}>\operatorname{deg} m_{x}=-1$, so that $f$ must vanish along some component of $C$, and we have seen that this is impossible.

Q.E.D.

Proof of (b). As discussed in Remark 2.2, the standard chain of reasoning is as follows:

1. $x \in C$ is a base point of $\left|K_{C}\right|$ if and only if $H^{0}\left(\mathcal{O}_{C}\left(K_{C}\right)\right) \rightarrow \mathcal{O}_{x}\left(K_{C}\right)$ is not onto, and then 
2. $H^{1}\left(m_{x} \mathcal{O}_{C}\left(K_{C}\right)\right) \rightarrow H^{1}\left(\mathcal{O}_{C}\left(K_{C}\right)\right)$ is not injective,

3. dually, $\operatorname{Hom}\left(\mathcal{O}_{C}\left(K_{C}\right), \omega_{C}\right) \rightarrow \operatorname{Hom}\left(m_{x} \mathcal{O}_{C}\left(K_{C}\right), \omega_{C}\right)$ is not onto,

4. therefore there exists a map $s: m_{x} \mathcal{O}_{C}\left(K_{C}\right) \rightarrow \omega_{C}$ linearly independent of the identity inclusion.

Now by Lemma 2.4, the map $s$ factors via an inclusion $m_{x} \mathcal{O}_{B}\left(K_{C}\right) \hookrightarrow$ $\omega_{B}$ on a generically Gorenstein curve $B$. But then $B \subsetneq C$ is forbidden by the numerically 2-connected assumption $\operatorname{deg} m_{x} \mathcal{O}_{B}\left(K_{C}\right)-\operatorname{deg} \omega_{B} \geq 1$.

Therefore $B=C$, that is, $s: m_{x} \mathcal{O}_{C}\left(K_{C}\right) \hookrightarrow \omega_{C}$ is an inclusion. After tensoring down with $-K_{C}$, this gives an inclusion $i: m_{x} \hookrightarrow \mathcal{O}_{C}$ linearly independent of the identity. Write $\mathcal{F}=i\left(m_{x}\right) \subset \mathcal{O}_{C}$. Then $\operatorname{deg} \mathcal{F}=-1$, and therefore $\mathcal{F}=m_{z}$ for some $z \in C$.

Now for any point $y \in C \backslash\{x\}$, there exists a linear combination $s^{\prime}=$ $s+\lambda$ id vanishing at $y$, which therefore defines an isomorphism $m_{x} \cong m_{y}$. This implies that every point $y \in C$ is a Cartier divisor, hence a nonsingular point. Since $C$ is clearly connected, and $\mathcal{O}_{C}(x-y) \cong \mathcal{O}_{C}$ for every $x, y \in C$, it follows that $C \cong \mathbb{P}^{1}$.

For the final statement, if $p_{a} C=0$ then $1=h^{0}\left(\mathcal{O}_{C}\right)=h^{1}\left(\omega_{C}\right)$ by (a) and duality, hence $h^{0}\left(\omega_{C}\right)=0$ by $\mathrm{RR}$, so that $H^{0}\left(\mathcal{O}_{C}\left(K_{C}\right)\right) \rightarrow \mathcal{O}_{x}$ is not onto for any $x \in C$.

Q.E.D.

Definition 3.4. We say that a Gorenstein curve $C$ is honestly hyperelliptic ([Ca1, Definition 3.18]) if there exists a finite morphism $\psi: C \rightarrow \mathbb{P}^{1}$ of degree 2 (that is, $\psi$ is finite and $\psi_{*} \mathcal{O}_{C}$ is locally free of rank 2 on $\mathbb{P}^{1}$ ). The linear system $\psi^{*}\left|\mathcal{O}_{C}(1)\right|$ defining $\psi$ is called an honest $g_{2}^{1}$.

We note the immediate consequences of the definition.

LEMMA 3.5. An honestly hyperelliptic curve $C$ of genus $p_{a} C=g \geq 0$ is isomorphic to a divisor $C_{2 g+2}$ in the weighted projective space $\mathbb{P}(1,1, g+1)$, not passing through the vertex $(0,0,1)$, defined by an equation

$$
w^{2}+a_{g+1}\left(x_{1}, x_{2}\right) w+b_{2 g+2}\left(x_{1}, x_{2}\right)=0 .
$$

It follows that every point of $C$ is either nonsingular or a planar double point, and that $C$ is either irreducible, or of the form $C=D_{1}+D_{2}$ with $D_{1} D_{2}=g+1$.

The projection $\varphi: C \rightarrow \mathbb{P}^{1}$ is a finite double cover, and the inverse image of any $x \in \mathbb{P}^{1}$ is a Cartier divisor which is a cluster $Z \subset C$ of degree 2. In other words, $Z$ is either 2 distinct nonsingular points of $C$, a nonsingular point with multiplicity 2 , or a section through a planar double point of $C$. 
THEOREM 3.6. Let $C$ be a numerically 3-connected Gorenstein curve. Then either $\left|K_{C}\right|$ is very ample or $C$ is honestly hyperelliptic.

In particular, in this case if $p_{a} C \geq 2$ then $K_{C}$ is ample, and if $p_{a} C=1$ then $C$ is honestly hyperelliptic.

Proof. Let $Z$ be a cluster of degree 2 for which $H^{0}\left(\mathcal{O}_{C}\left(K_{C}\right)\right) \rightarrow \mathcal{O}_{Z}\left(K_{C}\right)$ is not onto. The previous chain of reasoning gives a map $\mathcal{I}_{Z} \mathcal{O}_{C}\left(K_{C}\right) \rightarrow \omega_{C}$ linearly independent of the identity inclusion. An inclusion $\mathcal{I}_{Z} \mathcal{O}_{B}\left(K_{C}\right) \hookrightarrow$ $\omega_{B}$ with $B \subsetneq C$ is forbidden as before by $C$ numerically 3 -connected. Therefore we get an inclusion $s: \mathcal{I}_{Z} \mathcal{O}_{C}\left(K_{C}\right) \hookrightarrow \omega_{C}$ linearly independent of the identity inclusion. Note that any linear combination $s^{\prime}=s+\lambda$ id of the two sections is again generically injective, since an inclusion $\mathcal{I}_{Z} \mathcal{O}_{B}\left(K_{C}\right) \hookrightarrow \omega_{B}$ with $B \subsetneq C$ is forbidden by numerically 3 -connected.

The image $\mathcal{F}=s\left(\mathcal{I}_{Z} \mathcal{O}_{C}\left(K_{C}\right)\right) \subset \omega_{C}$ is a submodule of colength 2, therefore of the form $\mathcal{F}=\mathcal{I}_{Z^{\prime}} \omega_{C}$ for some cluster $Z^{\prime} \subset C$. Tensoring down the isomorphism $s: \mathcal{I}_{Z} \mathcal{O}_{C}\left(K_{C}\right) \rightarrow \mathcal{I}_{Z^{\prime}} \omega_{C}$ gives an isomorphism $s: \mathcal{I}_{Z} \cong \mathcal{I}_{Z^{\prime}}$, still linearly independent of the identity inclusion $\mathcal{I}_{Z} \hookrightarrow \mathcal{O}_{C}$.

Logically, there are 3 cases for $Z$ and $Z^{\prime}$. The first of these corresponds to an honest $g_{2}^{1}$ on $C$; the other two, corresponding to a $g_{2}^{1}$ with one or two base points, lead either to $p_{a} C \leq 1$ or to a contradiction. The case division is as follows:

Case $Z \cap Z^{\prime}=\emptyset$. Then the isomorphism $\mathcal{I}_{Z} \cong \mathcal{I}_{Z^{\prime}}$ implies that both $Z$ and $Z^{\prime}$ are Cartier divisors, and the two linearly independent inclusions $\mathcal{I}_{Z} \hookrightarrow \mathcal{O}_{C}$ define an honest $g_{2}^{1}$ on $C$. In more detail: $\mathcal{O}_{C}(Z)$ has 2 linearly independent sections with no common zeroes, and no linear combination of these vanishes on any component of $C$. Therefore $|Z|$ defines a finite 2-to-1 morphism $C \rightarrow \mathbb{P}^{1}$.

Case $Z=Z^{\prime}$. This case leads to an immediate contradiction. Indeed, take any point $x \notin \operatorname{Supp} Z$; then some linear combination of the two isomorphisms $s, \mathrm{id}: \mathcal{I}_{Z} \rightarrow \mathcal{I}_{Z}$ vanishes at $x$, and therefore vanishes along any reduced component of $C$ containing $x$. But we have just said that this is forbidden.

Case $Z \cap Z^{\prime}=x$. Here the case assumption can be rewritten $\mathcal{I}_{Z}+$ $\mathcal{I}_{Z^{\prime}}=m_{x}$. This case is substantial, and it really happens in two examples:

1. if $C$ is an irreducible plane cubic with a node or cusp $P$, and $Q, Q^{\prime} \in$ $C \backslash P$ then $m_{P} m_{Q} \cong m_{P} m_{Q^{\prime}} ;$ 
2. $\mathbb{P}^{1}$ has an incomplete $g_{2}^{1}$ with a fixed point, of the form $P+|Q|$.

We prove that we are in one of these cases. In either example, the curve $C$ has an honest $g_{2}^{1}$ (not directly given by our sections $s$, id), so the theorem is correct.

Claim 3.7. For any point $y \in C \backslash\{x\}$, there exists a linear combination $s^{\prime}=s+\lambda$ id defining an isomorphism $\mathcal{I}_{Z} \cong m_{x} m_{y}$.

Proof of Claim. Since $\mathcal{I}_{Z}, \mathcal{I}_{Z^{\prime}} \subset m_{x}$, we have two linearly independent maps $s$, id: $\mathcal{I}_{Z} \hookrightarrow m_{x}$, and some linear combination $s^{\prime}=s+\lambda$ id vanishes at $y$. Also, no map $\mathcal{I}_{Z} \rightarrow m_{x}$ vanishes along a component of $C$. Thus $s^{\prime}\left(\mathcal{I}_{Z}\right)=m_{x} m_{y}$.

Q.E.D.

It follows from the claim that $m_{x} m_{y} \cong m_{x} m_{y^{\prime}}$ for any two points $y, y^{\prime} \neq x$, so that $y, y^{\prime}$ are nonsingular, and $C$ is reduced and irreducible. Now let $\sigma: C_{1} \rightarrow C$ be the blow up of $m_{x}$. Then, essentially by definition of the blow up, $m_{x} \mathcal{O}_{C_{1}} \cong \mathcal{O}_{C_{1}}(-E)$ where $E$ is a Cartier divisor on $C_{1}$. Then $m_{C_{1}, y} \cong m_{C_{1}, y^{\prime}}$ for general points $y, y^{\prime} \in C_{1}$, hence as usual $C_{1} \cong \mathbb{P}^{1}$. If $C_{1} \cong C$ there is nothing more to prove.

If $C_{1} \not C$, the conductor ideal $\mathcal{C}=\mathcal{H} m_{\mathcal{O}_{C}}\left(\sigma_{*} \mathcal{O}_{C_{1}}, \mathcal{O}_{C}\right)$ of $\sigma_{*} \mathcal{O}_{C_{1}}$ in $\mathcal{O}_{C}$ is $m_{x}$. Indeed, let $f \in k(C)$ be the rational function such that multiplication by $f$ gives $m_{x} m_{y} \cong m_{x} m_{y^{\prime}}$; then $f$ is an affine parameter on $C_{1}=\mathbb{P}^{1}$ outside $y$, so that all regular functions on $C_{1}$ are regular functions of $f$, and $f m_{x}=m_{x}$ implies $\sigma_{*}\left(m_{x} \mathcal{O}_{C_{1}}\right)=m_{x} \subset \mathcal{O}_{C}$. Now it is known that the only Gorenstein curve singularity $x \in C$ with conductor ideal $m_{x}$ is a node or cusp (see [Se2, Chap. IV, §11] or [Re, Theorem 3.2]): indeed, $m_{x} \subset \mathcal{O}_{C} \subset$ $\sigma_{*} \mathcal{O}_{C_{1}}$, and the Gorenstein assumption $n=2 \delta$ gives length $\left(\sigma_{*} \mathcal{O}_{C_{1}} / \mathcal{O}_{C}\right)=$ $\operatorname{length}\left(\mathcal{O}_{C} / m_{x}\right)=1$. Therefore $p_{a} C=1$.

For the final statement, if $p_{a} C=1$ then $1=h^{0}\left(\mathcal{O}_{C}\right)=h^{1}\left(\omega_{C}\right)$ by Theorem 3.3, (a) and duality, hence $h^{0}\left(\omega_{C}\right)=1$ by RR, so that $H^{0}\left(\mathcal{O}_{C}\left(K_{C}\right)\right) \rightarrow$ $\mathcal{O}_{Z}$ is not onto for any cluster $Z \in C$ of degree 2 .

Q.E.D.

Remark 3.8. If $C$ is a numerically 3-connected Gorenstein curve with $p_{a} C \geq 2$, then Theorem 3.6 says that $K_{C}$ is automatically ample, and the usual dichotomy holds: either $K_{C}$ is very ample, or $C$ is honestly hyperelliptic.

Now assume instead that the dualising sheaf $\omega_{C}=\mathcal{O}_{C}\left(K_{C}\right)$ is ample and generated by its $H^{0}$. Equivalently, that $\left|K_{C}\right|$ is a free linear system, defining a finite morphism (the canonical morphism) $\varphi=\varphi_{K_{C}}: C \rightarrow \mathbb{P}^{p_{a}-1}$. 
In [Ca1], Definition 3.9, $C$ was defined to be hyperelliptic if $\varphi_{K_{C}}$ is not birational on some component of $C$. Thus by Theorem 3.6, in the 3 -connected case, hyperelliptic and honestly hyperelliptic coincide.

\section{$\S 4$. Canonical maps of surfaces of general type}

We give a slight refinement of a useful lemma due independently to J. Alexander and I. Bauer.

Lemma 4.1. (Alexander-Bauer) Suppose that $H$ is a Cartier divisor on an irreducible projective scheme $X$. Assume given effective Cartier divisors $D_{1}, D_{2}, D_{3}$ such that

(i) $H^{0}\left(\mathcal{O}_{X}(H)\right) \rightarrow H^{0}\left(\mathcal{O}_{D_{i}}(H)\right)$ is onto.

(ii) $H$ is very ample on every $\Delta \in\left|D_{i}\right|$ for $i=1,2,3$.

Then $H$ is very ample on $X$ if either

(a) $H \stackrel{\operatorname{lin}}{\sim} D_{1}+D_{2}$ and $\operatorname{dim}\left|D_{2}\right| \geq 1$, or

(b) $H \stackrel{\operatorname{lin}}{\sim} D_{1}+D_{2}+D_{3}$ and $\operatorname{dim}\left|D_{i}\right| \geq 1$ for $i=1,2,3$.

Proof. (a) is proved in [Ba1], Claim 2.19 and [Ra], Lemma 3.1, and also in [C-F], Prop. 5.1.

We prove (b). By Remark 2.1, we need to prove that if $x$ is any point of $X$, and $y$ is either another point of $X$ or a tangent vector at $x$, then $|H|$ separates $x$ from $y$. If some $\Delta_{i} \in\left|D_{i}\right|$ contains both $x$ and $y$, we are done by the assumptions (i) and (ii). In particular, since dim $\left|D_{i}\right| \geq 1$, such a $\Delta_{i}$ exists if $x$ or $y$ belong to the base locus of $\left|D_{i}\right|$.

Finally, if none of the above possibilities occurs, we can find $\Delta_{1}$ containing $x$ but not $y$, and $\Delta_{2}, \Delta_{3}$ containing neither $x$ nor $y$. Then $\Delta_{1}+\Delta_{2}+\Delta_{3}$ separates $x$ from $y$.

Q.E.D.

Proof of Theorem 1.2. Let $\pi: S \rightarrow X$ be the natural birational morphism from a minimal surface of general type $S$ to its canonical model $X$; write $K_{S}$ and $K_{X}$ for the canonical divisors of $S$ and $X$. Then $\omega_{X}$ is invertible and $\pi^{*}\left(\omega_{X}\right) \cong \omega_{S}$; in particular $H^{0}\left(X, m K_{X}\right) \cong H^{0}\left(X, m K_{S}\right)$ and $K_{X}^{2}=K_{S}^{2}$.

Step I. If $\left.C \in \mid(m-2) K_{X}\right) \mid$, then $H^{0}\left(\mathcal{O}_{X}\left(m K_{X}\right)\right) \rightarrow H^{0}\left(\mathcal{O}_{C}\left(m K_{X}\right)\right)$ is onto. This follows from our assumption $H^{1}\left(\mathcal{O}_{X}\left(2 K_{X}\right)\right)=0$. 
Step II. If $C \in\left|(m-2) K_{X}\right|$, then $\mathcal{O}_{C}\left(m K_{X}\right)$ is very ample.

Proof. By the curve embedding theorem Theorem 1.1, it is enough to prove that $m K_{X} B \geq 2 p_{a} B+1$ for every subcurve $B \subset C$. Note that by adjunction $K_{C}=(m-1) K_{X \mid C}$, so that we can write $m K_{X \mid B}=K_{X \mid B}+$ $K_{C \mid B}$. Since $K_{X}$ is ample, $K_{X} B \geq 1$, and therefore we need only prove that $K_{X} C \geq 3$ and

$$
\operatorname{deg} \mathcal{O}_{B}\left(K_{C}\right)-\operatorname{deg} \omega_{B} \geq 2 \text { for every strict subcurve } B \subset C,
$$

that is, that $C$ is numerically 2 -connected.

The corresponding fact for the minimal nonsingular model $S \rightarrow X$ is easy and well known ${ }^{1}$. Therefore $C$ numerically 2 -connected follows from the next result, whose proof we relegate to an appendix.

Lemma 4.2. Let $X$ be a surface with only Du Val singularities, and $\pi: S \rightarrow X$ the minimal resolution of singularities. Let $C \subset X$ be an effective Cartier divisor, and $C^{*}=\pi^{*} C$ the total transform of $C$ on $S$. Then

$$
C^{*} \text { numerically } k \text {-connected } \Longrightarrow \text { so is } C \text {. }
$$

Moreover, if $C^{*}$ is numerically 2-connected, and is only 3-disconnected by expressions $C^{*}=A+B$ where $A$ or $B$ is a-2-cycle exceptional for $\pi$, then $C$ is numerically 3 -connected.

Step III. $h^{0}\left((m-2) K_{X}\right) \geq 3$ if $m \geq 5$, and $\geq 2$ if $m=3$ or 4 .

Proof. For $m=3$ this is just the assumption $p_{g} \geq 2$.

For $m \geq 4$, if $p_{g} \geq 2$, then clearly $h^{0}\left((m-2) K_{X}\right) \geq 3$. Otherwise, in the case $p_{g} \leq 1$, we use the traditional numerical game of [B-M], based on Noether's formula $12 \chi\left(\mathcal{O}_{X}\right)=\left(c_{1}^{2}+c_{2}\right)(X)$. It consists of writing out Noether's formula using Betti numbers for the etale cohomology, in the form

$$
10+12 p_{g}=8 h^{1}\left(\mathcal{O}_{X}\right)+2 \Delta+b_{2}+K_{X}^{2} .
$$

\footnotetext{
${ }^{1}$ Tutorial: This is an easy consequence of the Hodge algebraic index theorem. If $D$ is nef and big and $D=A+B$ then $A^{2}+A B \geq 0, A B+B^{2} \geq 0$. The index theorem says that $A^{2} B^{2} \leq(A B)^{2}$, with equality only if $A, B$ are numerically equivalent to rational multiples of one another. The reader should carry out the easy exercise of seeing that $A B \leq 0$ gives a contradiction, and proving all the connected assertions we need. Or see [Bo, §4, Lemma 2] for details (the exceptional case $n=2,2 K_{S}=A+B$, with $A \stackrel{\text { num }}{\sim} B \stackrel{\text { num }}{\sim} K_{S}$ and $K_{S}^{2}=1$ is excluded by the assumption $K_{S}^{2} \geq 2$ if $m=4$ of Theorem 1.2).
} 
Here the nonclassical term $\Delta=2 h^{1}\left(\mathcal{O}_{X}\right)-b_{1}$ satisfies $\Delta \geq 0$, and $\Delta=0$ if char $k=0$. Since all the terms on the right hand side of (4) are $\geq 0$, it follows immediately that

$$
\begin{aligned}
& p_{g} \leq 1 \Longrightarrow h^{1}\left(\mathcal{O}_{X}\right) \leq 2 \\
& p_{g} \leq 0 \Longrightarrow h^{1}\left(\mathcal{O}_{X}\right) \leq 1
\end{aligned}
$$

Therefore, $p_{g} \leq 1$ implies $\chi\left(\mathcal{O}_{X}\right) \geq 0$; hence, for $m \geq 4$, by $\mathrm{RR}$

$$
h^{0}\left((m-2) K_{X}\right) \geq \chi\left(\mathcal{O}_{X}\right)+\left(\begin{array}{c}
m-2 \\
2
\end{array}\right) K_{X}^{2} \begin{cases}\geq 3 & \text { if } m \geq 5 \\
\geq 2 & \text { if } m=4\end{cases}
$$

Step IV. For $m=3$, we simply apply Lemma 4.1 , (b) to $3 K \stackrel{\operatorname{lin}}{\sim} K+$ $K+K$. For $m=4$ we apply Lemma 4.1, (a) to $4 K \stackrel{\operatorname{lin}}{\sim} 2 K+2 K$ : the assumptions (i) and (ii) of the lemma hold by Steps I, II and III.

For $m \geq 5$, we want to show that $H^{0}\left(\mathcal{O}_{X}\left(m K_{X}\right)\right) \rightarrow \mathcal{O}_{Z}$ is onto for any cluster $Z \subset X$ of degree 2 . But by Step III, there exists $C \in\left|(m-2) K_{X}\right|$ containing $Z$. The result then follows by Steps I and II.

Q.E.D.

\section{Appendix: Proof of Lemma 4.2}

Suppose that $B \subset C$ is a strict subcurve. Write $B^{\prime}$ for the birational (= strict or proper) transform of $B$ in $S$ and $C^{*}=\pi^{*} C$ for the total transform of $C$. For the proof, we find a divisor $\widehat{B}$ (the hat transform) with the properties

(i) $B^{\prime} \leq \widehat{B} \leq C^{*}$ and $\widehat{B}-B^{\prime}$ contains only exceptional curves;

(ii) $p_{a} \widehat{B}=p_{a} B$.

Suppose first that we know $\widehat{B}$ satisfying these conditions. Then

$$
\left(C^{*}-\widehat{B}\right) \widehat{B} \geq k
$$

by the assumption on $C^{*}$, which we write

$$
\left(K_{S}+C^{*}\right) \widehat{B}-\left(K_{S}+\widehat{B}\right) \widehat{B} \geq k .
$$

Here the first term equals $\left(K_{X}+C\right) B=\operatorname{deg} \mathcal{O}_{B}\left(K_{C}\right)$, and the second $2 p_{a} \widehat{B}-2=2 p_{a} B-2$. Thus

$$
\operatorname{deg} \mathcal{O}_{B}\left(K_{C}\right)-\left(2 p_{a} B-2\right)=\left(K_{S}+C^{*}\right) \widehat{B}-\left(2 p_{a} \widehat{B}-2\right) \geq k .
$$


So it is enough to find $\widehat{B}$. For this, following the methods of [Ar1][Ar2], let $\left\{\Gamma_{i}\right\}$ be all the exceptional -2-curves. Define $\widehat{B}=B^{\prime}+\sum e_{i} \Gamma_{i}$ with $e_{i} \in \mathbb{Z}, e_{i} \geq 0$ minimal with respect to the property $\widehat{B} \Gamma_{i} \leq 0$; this exists, because $C^{*}-A^{\prime}$ has the stated property (where $A^{\prime}$ is the birational transform of the residual Weil divisor $C-B)$.

Claim 4.3. The curve $\widehat{B}$ has the following properties:

(iii) $\omega_{B}=\pi_{*} \omega_{\widehat{B}}$;

(iv) $R^{1} \pi_{*} \omega_{\widehat{B}}=0$.

Therefore $p_{a} \widehat{B}=p_{a} B$.

Proof of Claim. Taking $\pi_{*}$ of the short exact sequence

$$
0 \longrightarrow \mathcal{O}_{S}\left(K_{S}\right) \longrightarrow \mathcal{O}_{S}\left(K_{S}+\widehat{B}\right) \longrightarrow \omega_{\widehat{B}} \longrightarrow 0
$$

gives $0 \rightarrow \mathcal{O}_{X}\left(K_{X}\right) \rightarrow \mathcal{O}_{X}\left(K_{X}+B\right) \rightarrow \pi_{*} \omega_{\widehat{B}} \rightarrow 0$ and $R^{1} \pi_{*} \mathcal{O}_{S}\left(K_{S}+\widehat{B}\right)=$ $R^{1} \pi_{*} \omega_{\widehat{B}}$. The first of these implies that $\omega_{B}=\pi_{*} \omega_{\widehat{B}}$. Indeed, if $B \subset X$ is an effective Weil divisor on any Cohen-Macaulay variety then the adjunction formula $\omega_{B}=\mathcal{E} x t_{\mathcal{O}_{X}}^{1}\left(\mathcal{O}_{B}, \omega_{X}\right)$ (see, for example, [Re, Theorem 2.12, (1)]) boils down to an exact sequence $0 \rightarrow \mathcal{O}_{X}\left(K_{X}\right) \rightarrow \mathcal{O}_{X}\left(K_{X}+B\right) \rightarrow \omega_{B} \rightarrow 0$. This proves (iii).

By the method of $[\operatorname{Ar} 1]-[\operatorname{Ar} 2]$,

$$
R^{1} \pi_{*} \mathcal{O}_{S}\left(K_{S}+\widehat{B}\right)=\varliminf_{\lim } H^{1}\left(D, \mathcal{O}_{D}\left(K_{S}+\widehat{B}\right)\right),
$$

where the inverse limit is taken over effective divisors $D=\sum a_{j} \Gamma_{j}$. If all the $H^{1}=0$, the limit is zero, as required.

Suppose then by contradiction that $D=\sum a_{j} \Gamma_{j}$ has $H^{1}\left(\mathcal{O}_{D}\left(K_{S}+\widehat{B}\right)\right)$ $\neq 0$. Then dually, $\operatorname{Hom}\left(\mathcal{O}_{D}\left(K_{S}+\widehat{B}\right), \omega_{D}\right) \neq 0$, and Lemma 2.4 gives an inclusion $\mathcal{O}_{D}\left(K_{S}+\widehat{B}\right) \hookrightarrow \omega_{D}$ (for a possibly smaller $D$ ). Writing out the adjunction formula for $\omega_{D}$ and tensoring down by $K_{S}+\widehat{B}$ gives $\mathcal{O}_{D} \hookrightarrow$ $\mathcal{O}_{D}(D-\widehat{B})$. Therefore $(\widehat{B}-D) \Gamma_{i} \leq 0$ for every $\Gamma_{i} \subset D$, and by construction of $\widehat{B}$ for the other $\Gamma_{i}$. Now $\widehat{B}-D=B^{\prime}+\sum e_{j}^{\prime} \Gamma_{j}$ contradicts the minimality of $\widehat{B}$, provided we show that the $e_{j}^{\prime} \geq 0$. For this, note that

$$
\left(\sum e_{j}^{\prime} \Gamma_{j}\right) \Gamma_{i}=(\widehat{B}-D) \Gamma_{i}-B^{\prime} \Gamma_{i} \leq 0 \quad \text { for every } i
$$

and the intersection form on the $\Gamma_{i}$ is negative definite, so that the standard argument implies $\sum e_{j}^{\prime} \Gamma_{j} \geq 0$ (write it as $A-B$ where $A, B \geq 0$ have no common divisor, and calculate $\left.B^{2}\right)$.

Q.E.D. 


\section{$\S 5$. The tricanonical map}

We state the following points as independent lemmas in order to tidy up our proofs, and because they might be useful elsewhere. The first is a particular case of the numerical criterion for flatness, see [Ha, Chap. III, Theorem 9.9].

LEMMA 5.1. (Flat double covers) If $\varphi: X \rightarrow Y$ is a generically 2to-1 morphism (say with $Y$ integral), then for any $y \in Y$, the condition length $\varphi^{-1}(y)=2$ implies that $\varphi$ is flat over a neighbourhood of $y$.

LEMmA 5.2. (Class group) Let $X$ be a normal surface; then the functor of Weil divisors on $X$ modulo linear equivalence is represented by a group scheme $\mathrm{WCl} X$ which is the product of a finitely generated Abelian group by a group scheme of finite type.

Proof. Let $Y \rightarrow X$ be a resolution, and write $Y_{0}=Y \backslash$ exceptional locus $\cong X \backslash \operatorname{Sing} X$. Then obviously $\mathrm{WCl} X=\operatorname{Pic} Y_{0}$ and $\operatorname{Pic} Y \rightarrow \operatorname{Pic} Y_{0}$ is surjective (on the level of functors).

Q.E.D.

Lemma 5.3. (Push-down of invariant linear systems) Let $\varphi: X \rightarrow Y$ be a finite morphism of degree 2, where $X$ and $Y$ are normal. Suppose that $L$ is a linear system of Cartier divisors on $X$ with the property that $\varphi_{\mid D}: D \rightarrow \Gamma_{D}=\varphi(D)$ has degree 2 for every $D \in L$. Then the $\Gamma_{D}$ are linearly equivalent Weil divisors, that is, they are all members of one linear system.

Proof. For any $D, D^{\prime} \in L$, note that $2 \Gamma_{D}=\pi_{*} D$ is a Cartier divisor on $Y$, and $2 \Gamma_{D} \stackrel{\operatorname{lin}}{\sim} 2 \Gamma_{D^{\prime}}$, because if $D$ is locally defined by $f \in k(X)$ (or $D-D^{\prime}=\operatorname{div} f$ ) then $2 \Gamma_{D}$ is locally defined by $\operatorname{Norm}(f)$, where Norm $=$ $\operatorname{Norm}_{k(X) / k(Y)}$.

Thus the Weil divisor class $\Gamma_{D}-\Gamma_{D^{\prime}}$ is a 2-torsion element of the Weil divisor class group $\mathrm{WCl} Y$, which by Lemma 5.2 is an algebraic group of finite type. Its 2-torsion subgroup is a finite algebraic group scheme $G$. Now for fixed $D_{0} \in L$, taking $D \mapsto \Gamma_{D}-\Gamma_{D_{0}}$ defines a morphism from the parameter space of the linear system $L$ to $G$, which must be the constant morphism to 0 . This proves what we need.

Assuming that $\varphi$ is separable make this argument more intuitive, since then it is Galois, and $\varphi_{*} \mathcal{O}_{X}$ splits into invariant and antiinvariant parts: $\varphi_{*} \mathcal{O}_{X}=\mathcal{O}_{Y} \oplus \mathcal{L}$, with $\mathcal{L}$ a divisorial sheaf. Then $\Gamma_{D}$ is locally either a 
Cartier divisor or in the local Weil divisor class of $\mathcal{L}$, and $\Gamma_{D}-\Gamma_{D^{\prime}}$ is in the kernel of $\varphi^{*}$, which is a finite algebraic group scheme, etc.

Q.E.D.

LEMMA 5.4. Let $\Lambda$ be a linear system of Weil divisors through a point $P$ on a normal surface $Y$. Then the curves in $\Lambda$ singular at $P$ form a projective linear subspace of codimension $\leq 2$.

Proof. Easy exercise involving the resolution and birational transform.

Q.E.D.

Proof of Theorem 1.3, Case (a). Since $q^{\prime}=0$, we have $\chi\left(\mathcal{O}_{X}\right) \geq 1$, and $K_{X}^{2} \geq 3$ gives $P_{2}=h^{0}\left(2 K_{X}\right) \geq 4$. Let $Z$ be a cluster of degree 2 on $X$. Since $P_{2} \geq 4$, the linear subsystem $\left|2 K_{X}-Z\right|$ consisting of curves $D \in\left|2 K_{X}\right|$ through $Z$ has dimension $\geq 1$, and any $D \in\left|2 K_{X}\right|$ is 3 -connected by the final part of Lemma 4.2 (whose assumptions are easily verified as in [Bo, $\S 4$, Lemma 2]). By $H^{1}\left(K_{X}\right)=0$, the sequence

$$
0 \longrightarrow H^{0}\left(X, \mathcal{O}_{X}\left(K_{X}\right)\right) \longrightarrow H^{0}\left(X, \mathcal{O}_{X}\left(3 K_{X}\right)\right) \longrightarrow H^{0}\left(D, \omega_{D}\right) \longrightarrow 0
$$

is exact. Since $\left|\omega_{D}\right|$ is free by Theorem 3.3 , it follows that $\varphi=\varphi_{3 K_{X}}$ is a finite morphism $\varphi: X \rightarrow Y \subset \mathbb{P}^{N}$, where $N=P_{3}-1$. Assume that $\left|3 K_{X}\right|$ does not separate $Z$. Then, by Theorem 3.6, $D$ is honestly hyperelliptic. Since the same argument applies to any $D \in\left|2 K_{X}-Z\right|$, it follows that $\operatorname{deg} \varphi \geq 2$.

On the other hand, for any point $y \in Y$, if the scheme theoretic fibre $\varphi^{-1}(y)$ is a cluster of degree $\geq 3$, then there is a curve $D^{\prime} \in\left|2 K_{X}\right|$ containing $\varphi^{-1}(y)$, and $\varphi^{-1}(y)$ is contained in a fibre of $\varphi_{\omega_{D^{\prime}}}: D^{\prime} \rightarrow \mathbb{P}^{1}$, which contradicts Lemma 3.5. Hence $\varphi: X \rightarrow Y$ is of degree 2 (possibly inseparable if char $k=2$ ). In particular $2 \mid 9 K^{2}$, so that $K^{2}$ is even and $K^{2} \geq 4$; thus $P_{2} \geq 5$, and $\operatorname{dim}\left|2 K_{X}-Z\right| \geq 2$ for any cluster $Z$ of degree 2 . By changing $Z$ if necessary, we can assume that $\varphi(Z)=y \in Y$ is a general point, and is thus nonsingular. We have just shown that every fibre $\varphi^{-1}(y)$ has degree exactly 2 , so that $\varphi$ is flat by Lemma 5.1 ; it is easy to see that this implies that $Y$ is normal.

Now for any $D \in\left|2 K_{X}-Z\right|$, the image $\varphi(D)=\Gamma_{D} \subset Y$ is a curve through $y=\varphi(Z)$ isomorphic to $\mathbb{P}^{1}$, and $\operatorname{deg} \varphi_{\mid D}=\operatorname{deg} \varphi=2$. By Lemma 5.3 the $\Gamma_{D} \subset Y$ are linearly equivalent, so that they are all contained in a linear system. This contradicts Lemma 5.4: in any linear system of curves through $y$, curves singular at $y$ form a linear subsystem of codimension $\leq 2$, whereas 
the $\Gamma_{D}$ for $D \in\left|2 K_{X}-Z\right|$ form an algebraic subfamily of nonsingular curves depending with a complete parameter space of dimension $\geq 2$ made up of curves isomorphic to $\mathbb{P}^{1}$.

Q.E.D.

Remark 5.5. Here we have assumed that $\varphi(Z)=y \in Y$ is a general point only for simplicity (see Lemma 5.4).

Proof of Theorem 1.3, Case (b). Let $Z$ be a cluster of degree 2 on $X$ and $x \in Z$ a reduced point; that is, $Z$ is either a pair $(x, y)$ of distinct points, or a point $x$ plus a tangent vector $y$ at $x$. We assume that $\left|3 K_{X}\right|$ does not separate $Z$, and gather together a number of deductions concerning the curves

$$
C_{L} \in\left|K_{X}+L\right| \text { and } D_{L} \in\left|2 K_{X}-L\right| \text { for all } L \in \operatorname{Pic}^{0} X
$$

arriving eventually at a contradiction.

Step A. $h^{0}\left(K_{X}+L\right) \geq 1$ for all $L \in \operatorname{Pic}^{0} X$. In fact if $L \neq 0$ then $h^{2}\left(K_{X}+L\right)=0$, and hence $h^{0}\left(K_{X}+L\right) \geq \chi\left(K_{X}\right) \geq 1$.

Step B. $Z \not \subset C_{L}$ for all $L \in \operatorname{Pic}^{0} X$ and all $C_{L} \in\left|K_{X}+L\right|$. Indeed

$$
H^{0}\left(X, \mathcal{O}_{X}(3 K)\right) \longrightarrow H^{0}\left(C_{L}, \mathcal{O}_{C_{L}}\left(3 K_{X}\right)\right)
$$

is onto by the assumption $H^{1}\left(\mathcal{O}_{X}\left(2 K_{X}-L\right)\right)=0$, and $\mathcal{O}_{C_{L}}\left(3 K_{X}\right)$ very ample follows from Theorem 1.1 exactly as in $\S 4$, Step II. Therefore if $Z \subset$ $C_{L}$ then $\left|3 K_{X}\right|$ separates $Z$, which we are assuming is not the case.

Step C. For general $L \in \operatorname{Pic}^{0} X$ and all $C_{L} \in\left|K_{X}+L\right|$ we have $x \in C_{L}$.

First of all, since $\operatorname{dim} \operatorname{Pic}^{0} X \geq 1$, there is an $L \in \operatorname{Pic}^{0} X$ and a curve $C_{L} \in\left|K_{X}+L\right|$ containing $x$, and $C_{L}$ does not contain $Z$ by Step B. Now if $L_{1}, L_{2} \in \operatorname{Pic}^{0} X$ is a general solution of $L+L_{1}+L_{2}=0$, and $x \notin C_{L_{1}}$, $x \notin C_{L_{2}}$, then $C_{L}+C_{L_{1}}+C_{L_{2}}$ separates $x$ and $Z$, a contradiction.

Step D. $h^{0}\left(K_{X}+L\right)=1$ and $H^{1}\left(K_{X}+L\right)=0$ for general $L \in \operatorname{Pic}^{0} X$.

By Step C, every $s \in H^{0}\left(K_{X}+L\right)$ vanishes at $x$. If $h^{0}\left(K_{X}+L\right) \geq 2$ then some nonzero section would vanish also at $y$. The statement about $H^{1}$ follows from RR:

$$
1=h^{0}\left(\mathcal{O}_{X}\left(K_{X}+L\right)\right) \geq \chi\left(\mathcal{O}_{X}\left(K_{X}+L\right)\right)=\chi\left(\mathcal{O}_{X}\right) \geq 1
$$


Step E. $x \in \operatorname{Bs}\left|2 K_{X}-L\right|$ for general $L \in \operatorname{Pic}^{0} X$. For if $D_{L} \in$ $\left|2 K_{X}-L\right|$ does not contain $x$ then $D_{L}+C_{L}$ separates $x$ from $Z$ (since by Step B already $C_{L}$ separates them).

Step F. For general $L, L_{1} \in \operatorname{Pic}^{0} X$, the point $x$ is a base point of the linear system $\left|\left(2 K_{X}-L_{1}\right)_{\mid C_{L}}\right|$ on $C_{L}$, and hence

$$
H^{1}\left(m_{x} \mathcal{O}_{C_{L}}\left(2 K_{X}-L_{1}\right)\right) \neq 0 \text {. }
$$

This follows from $x \in \operatorname{Bs}\left|2 K_{X}-L_{1}\right|$ because by Step D, restriction from $X$ maps onto $H^{0}\left(\mathcal{O}_{C_{L}}\left(2 K_{X}-L_{1}\right)\right)$.

Step G. We now observe that Step B implies that $x$ is a singular point of $C_{L}$. If $x \in \operatorname{Sing} X$ then it is automatically singular on $C_{L}$. On the other hand, if $x$ is nonsingular on $X$ and on $C_{L}$, consider the blowup $\sigma: X_{1} \rightarrow X$ of $x$ and the algebraic system $C_{L}^{\prime}=\sigma^{*} C_{L}-E$, where $E$ is the exceptional divisor. Let $y \in X_{1}$ be the point corresponding either to the other point or to the tangent vector of the cluster $Z$. Since the curves $C_{L}^{\prime}$ move in a positive dimensional system, there is a curve $C_{L}^{\prime}$ through $y$, and therefore a curve $C_{L}$ containing $Z$, contradicting Step B.

Step H. For general elements $L, L_{2} \in \mathrm{Pic}^{0} X$, there is an isomorphism $m_{x} \mathcal{O}_{C_{L}}\left(L_{2}\right) \cong m_{x}$.

This follows as usual by automatic adjunction (Lemma 2.4) applied to the conclusion $H^{1}\left(m_{x} \mathcal{O}_{C_{L}}\left(2 K_{X}-L_{1}\right)\right) \neq 0$ of Step F, where $L_{1}=-L-L_{2}$. We first get a nonzero homomorphism

$$
m_{x} \mathcal{O}_{C_{L}}\left(2 K_{X}-L_{1}\right) \longrightarrow \omega_{C_{L}}=\mathcal{O}_{C_{L}}\left(2 K_{X}+L\right),
$$

that is, a map $m_{x} \mathcal{O}_{C_{L}}\left(L_{2}\right) \rightarrow \mathcal{O}_{C_{L}}$; since $C_{L}$ is 2-connected this must be an inclusion, and the image is the ideal of a point $m_{z}$. But $x$ is a singular point of $C_{L}$ (by Step $\mathrm{G}$ ), and thus $x=z$.

Step I. Let $\sigma: C^{\prime} \rightarrow C=C_{L}$ be the blowup at $x$. Step $\mathrm{H}$ implies that $L_{2}^{\prime}=\sigma^{*} L_{2}$ is trivial on $C^{\prime}$ for every general $L_{2}$, and hence for every $L_{2} \in$ $\mathrm{Pic}^{0} X$ (by the group law). We derive a contradiction from this. Consider the diagram

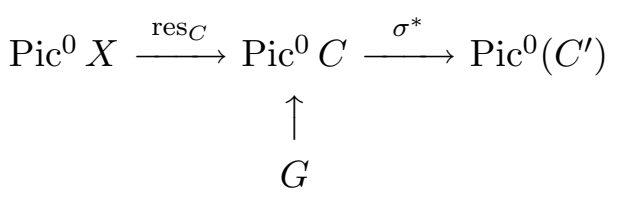


where $G$ is the kernel of $\sigma^{*}$. Now the key point (exactly as in Ramanujam and Francia vanishing) is that $G$ is an affine group scheme. Since the composite $\sigma^{*} \circ \operatorname{res}_{C}$ is zero, $\operatorname{Pic}^{0} X$ maps to $G$. Since $\operatorname{Pic}^{0} X$ is complete res $C$ is the constant morphism to zero. But this is obviously nonsense: for example, since $H^{1}\left(\mathcal{O}_{X}\left(2 K_{X}+L\right)\right)=0$ for all $L \in \operatorname{Pic}^{0} X$, the exact sequence

$$
0 \longrightarrow \mathcal{O}_{X}\left(-K_{X}-L+N\right) \longrightarrow \mathcal{O}_{X}(N) \longrightarrow \mathcal{O}_{C}(N) \longrightarrow 0
$$

is exact on global sections if $L \neq N$. Thus $H^{0}\left(\mathcal{O}_{C}(N)\right)=0$ and the restriction of $N$ to $C$ is nontrivial.

Q.E.D.

\section{$\S 6$. The bicanonical map}

\section{Preliminaries and the proof of Theorem 1.4, (a) and (c)}

This section proves Theorem 1.4. We start by remarking that $\left|2 K_{X}\right|$ is free. Indeed, for any $C \in\left|K_{X}\right|$, the restriction $\mathcal{O}_{X}\left(2 K_{X}\right) \rightarrow \mathcal{O}_{C}\left(K_{C}\right)$ is surjective on $H^{0}$, and $\left|K_{C}\right|$ is free by Theorem 3.3. For a cluster $Z$ of degree 2 in $X$, note the following obvious facts:

(i) If $Z$ is contracted by $\left|2 K_{X}\right|$ then $\left|K_{X}\right|$ does not separate $Z$; thus

$$
h^{0}\left(\mathcal{I}_{Z} \mathcal{O}_{X}\left(K_{X}\right)\right) \geq p_{g}-1 \text { or } \operatorname{dim}\left|K_{X}-Z\right| \geq p_{g}-2 .
$$

(ii) If $\left|2 K_{X}\right|$ contracts $Z$ then so does $\left|K_{C}\right|$ for any curve $C \in\left|K_{X}-Z\right|$.

Proof of Theorem 1.4, (a). We suppose that every curve $C \in\left|K_{X}-Z\right|$ is 3-connected, and derive a contradiction from the assumption that $\left|2 K_{X}\right|$ contracts $Z$. By Theorem 3.6, every $C \in\left|K_{X}-Z\right|$ is honestly hyperelliptic. As in the proof of Theorem 1.3, Case (a), it follows that $\varphi_{2 K}: X \rightarrow Y$ has degree 2 , and maps every $C \in\left|K_{X}-Z\right|$ as a double cover of a curve $\Gamma_{C} \subset Y$ isomorphic to $\mathbb{P}^{1}$. Then $\Gamma_{C}$ for $C \in\left|K_{X}-Z\right|$ form an algebraic subfamily of a linear system of curves through $y=\varphi_{2 K}(Z)$, with a complete parameter space of dimension $\geq 2$. As before, this contradicts Lemma 5.4 (but $y \in Y$ may now be singular).

Q.E.D.

Definition 6.1. Let $X$ be a projective surface with at worst $\mathrm{Du}$ Val singularities and with $K_{X}$ nef. A Francia curve or Francia cycle is an effective Weil divisor $B$ on $X$ satisfying

$$
K_{X} B=p_{a} B=1 \text { or } 2 .
$$


If $K_{X}$ is ample and $B$ is Gorenstein (for example if $B$ is a Cartier divisor), it is clearly either an irreducible curve of genus 1 , or a numerically 2-connected curve of arithmetic genus $p_{a}=2$. It would be interesting to know if $B$ is necessarily Gorenstein.

Proof of Theorem 1.4, (b) $\Longrightarrow(\mathrm{c})$. The argument is standard and we omit some details. Suppose that the 2-canonical map $\varphi=\varphi_{2 K}: X \rightarrow Y$ is not birational. Every point $x \in X$ is contained in a cluster $Z$ of degree 2 contracted by $\varphi$; we choose $x \in$ NonSing $X$. Theorem 1.4, (b) gives a Francia curve $B_{0} \subset X$ through $Z$. Write $S \rightarrow X$ for the minimal nonsingular model of $X$ and $B=\widehat{B}_{0}$ for the hat transform of $B_{0}$ (as in the proof of Lemma 4.2). Then by Claim $4.3, B$ is also a Francia cycle on $S$, that is, $1 \leq K_{S} B=p_{a} B \leq 2$. An easy argument in quadratic forms shows that there are at most finitely many effective divisors $B \subset S$ with $K_{S} B=1$ and $B^{2}=-1$ (compare [Bo, pp. 191-192] or [B-P-V, p. 224]). Therefore every general point of $S$ is contained in a curve $B$ with $K_{S} B=p_{a} B=2$, and hence $B^{2}=0$. Now the same argument in quadratic forms shows that divisors with $K_{S} B=2$ and $B^{2}=0$ belong to finitely many numerical equivalence classes, so one class must contain an algebraic family of curves. This gives a genus 2 pencil on $S$, and therefore also on $X$.

Q.E.D.

We use the following obvious lemma at several points in what follows.

LEMma 6.2. (Dimension lemma) Let $\eta \subset X$ be a cluster of degree $d$ which is contracted by $\left|2 K_{X}\right|$, and $C \in\left|K_{X}\right|$ a curve containing $\eta$. Then

$$
h^{1}\left(\mathcal{I}_{\eta} \mathcal{O}_{C}\left(K_{C}\right)\right)=\operatorname{dim} \operatorname{Hom}\left(\mathcal{I}_{\eta}, \mathcal{O}_{C}\right)=d
$$

In particular, for any $x \in C$, we have

$$
h^{1}\left(m_{x}^{2} \mathcal{O}_{C}\left(K_{C}\right)\right)=\operatorname{dim} \operatorname{Hom}\left(m_{x}^{2} \mathcal{O}_{C}, \mathcal{O}_{C}\right)=1+\operatorname{dim} T_{\varphi, x} \leq 4,
$$

where $T_{\varphi, x}$ is the Zariski tangent space to the scheme theoretic fibre of $\varphi_{2 K_{X}}$ through $x$.

Proof. Since $\left|K_{C}\right|$ is free and contracts $\eta$, the evaluation map $H^{0}\left(\mathcal{O}_{C}\left(K_{C}\right)\right) \rightarrow \mathcal{O}_{\eta}\left(K_{C}\right)=k^{d}$ has rank 1 , so that $h^{1}\left(\mathcal{I}_{\eta} \mathcal{O}_{C}\left(K_{C}\right)\right)=d$ comes from the exact sequence

$$
\begin{gathered}
0 \longrightarrow H^{0}\left(\mathcal{I}_{\eta} \mathcal{O}_{C}\left(K_{C}\right)\right) \longrightarrow H^{0}\left(\mathcal{O}_{C}\left(K_{C}\right)\right) \longrightarrow k^{d} \\
\longrightarrow H^{1}\left(\mathcal{I}_{\eta} \mathcal{O}_{C}\left(K_{C}\right)\right) \longrightarrow H^{1}\left(\mathcal{O}_{C}\left(K_{C}\right)\right)=k
\end{gathered}
$$


As usual, Serre duality gives

$$
\operatorname{Hom}\left(\mathcal{I}_{\eta}, \mathcal{O}_{C}\right)=\operatorname{Hom}\left(\mathcal{I}_{\eta} \mathcal{O}_{C}\left(K_{C}\right), \omega_{C}\right) \stackrel{\mathrm{d}}{ } H^{1}\left(\mathcal{I}_{\eta} \mathcal{O}_{C}\left(K_{C}\right)\right)
$$

We obtain the last part by taking $\eta$ to be the intersection of the scheme theoretic fibre $\varphi^{-1}(\varphi(x))$ with the subscheme $V\left(m_{x}^{2}\right) \subset C$ corresponding to the tangent space.

Q.E.D.

\section{Case division and plan of proof of (b)}

Throughout this section, $Z$ is a cluster of degree 2, and we argue by restricting to a curve $C \in\left|K_{X}-Z\right|$, usually imposing singularities on $C$ at a point $x \in Z$. As usual, the assumption that $Z$ is contracted by $K_{C}$ gives a homomorphism $\mathcal{I}_{Z} \rightarrow \mathcal{O}_{C}$ linearly independent of the identity inclusion. By passing to a suitable linear combination $s^{\prime}=s+\lambda$ id if necessary, we assume that $s \in \operatorname{Hom}\left(\mathcal{I}_{Z}, \mathcal{O}_{C}\right)$ is injective, and hence $s\left(\mathcal{I}_{Z}\right)=\mathcal{I}_{Z^{\prime}}$ for some cluster $Z^{\prime}$ of degree 2 ; the family of clusters $Z^{\prime}$ as $s$ runs through injective elements $s \in \operatorname{Hom}\left(\mathcal{I}_{Z}, \mathcal{O}_{C}\right)$ is an analog of a $g_{2}^{1}$ on $C$.

The argument is modelled on the proof of Theorem 3.6. As there, we use different arguments depending on how $Z$ and $Z^{\prime}$ intersect, or, to put it another way, how $Z^{\prime}$ moves as $s$ runs through injective elements $s \in$ $\operatorname{Hom}\left(\mathcal{I}_{Z}, \mathcal{O}_{C}\right)$. (In other words, how the $g_{2}^{1}$ corresponding to $\operatorname{Hom}\left(\mathcal{I}_{Z}, \mathcal{O}_{C}\right)$ breaks up into a "base locus" plus a "moving part".) Let $s \in \operatorname{Hom}\left(\mathcal{I}_{Z}, \mathcal{O}_{C}\right)$ be a general element, and $\mathcal{I}_{Z^{\prime}}=s\left(\mathcal{I}_{Z}\right)$. Logically, there are 4 cases for $Z$ and $Z^{\prime}$.

1. $\operatorname{Supp} Z \cap \operatorname{Supp} Z^{\prime}=\emptyset$.

2. $\operatorname{Supp} Z \cap \operatorname{Supp} Z^{\prime} \neq \emptyset$, but $\operatorname{Supp} Z \neq \operatorname{Supp} Z^{\prime}$.

3. $Z=Z^{\prime}$.

4. $Z \neq Z^{\prime}$ are nonreduced clusters supported at the same point $x \in X$.

In Case 2, $|Z|$ has a fixed point plus a moving point; as we see in Lemma 6.4 , this contradicts $K_{X}$ ample. In Case $1,|Z|$ is a free $g_{2}^{1}$, and the isomorphism $\mathcal{I}_{Z} \cong \mathcal{I}_{Z^{\prime}}$ with $\operatorname{Supp} Z \cap \operatorname{Supp} Z^{\prime}=\emptyset$ implies that $\mathcal{I}_{Z}$ is locally free, so that $Z$ is a Cartier divisor on $C$. If $p_{g} \geq 4$, it turns out that we can choose $C$ to be "sufficiently singular" at a point $x \in Z$ so that $Z \subset C$ is not Cartier, and Case 1 is excluded for such $C$ (see Lemma 6.5).

In Cases 3-4, when the support of $Z$ does not move, we must find a map $s^{\prime}: \mathcal{I}_{Z} \rightarrow \mathcal{O}_{C}$ vanishing on a "fairly large" portion of $C$, so that its 
scheme theoretic support $B \subset C$ is "fairly small". The key idea is to look for $s^{\prime}$ as a nilpotent or idempotent (see Lemma 6.7 and Corollary 6.8). The assumption of Case 3 is $\operatorname{Hom}\left(\mathcal{I}_{Z}, \mathcal{O}_{C}\right)=\operatorname{End}\left(\mathcal{I}_{Z}\right)$, which is a 2-dimensional Artinian algebra; this makes it is rather easy to find a nilpotent or idempotent element, and to prove Theorem 1.4, (b).

In Case $4, Z^{\prime}$ is $x$ plus a tangent vector $y$ which moves in $T_{C, x}$ as $s \in \operatorname{Hom}\left(\mathcal{I}_{Z}, \mathcal{O}_{C}\right)$ runs through injective elements; this is an infinitesimal $g_{2}^{1}$, an interesting geometric phenomenon in its own right (see Remark 6.3 and the proof of Proposition 6.9, Step 6 for more details). The key point in this case is to prove that the extra homomorphism $s: \mathcal{I}_{Z} \rightarrow \mathcal{O}_{C}$ takes $m_{x}^{2}$ to itself, so that $\operatorname{End}\left(m_{x}^{2}\right)$ is a nontrivial Artinian algebra; see Proposition 6.9.

Remark 6.3. In Case 4, reversing the usual argument proves that $\varphi_{K_{C}}$ also contracts $Z^{\prime}$, and so it contracts a cluster $\eta$ of degree $\geq 3$ contained in the first order tangent scheme $V\left(m_{x}^{2}\right) \subset C$. If $C$ is numerically 3-connected, this is of course impossible by Theorem 3.6. In this case, $\operatorname{Hom}\left(\mathcal{I}_{\eta}, \mathcal{O}_{C}\right)$ is a certain analog of a $g_{3}^{2}$ or $g_{4}^{3}$ on $C$.

Case 4 certainly happens on abstract numerically 2-connected Gorenstein curves, and more generally, the analog of a $g_{m}^{m-1}$. Example: let $C_{i}$ for $i=1, \ldots, m$ be nonhyperelliptic curves of genus $g_{i} \geq 3$ with marked points $x_{i} \in C_{i}$, and assemble the $C_{i}$ into a curve $C=\bigcup C_{i}$ by glueing together all the $x_{i}$ to one point $x$, at which the tangent directions are subject to a single nondegenerate linear relation, so that the singularity $x \in C$ is analytically equivalent to the cone over a frame of reference $\left\{P_{1}, P_{2}, \ldots, P_{m}\right\}$ in $\mathbb{P}^{m-2}$. Then $C$ is Gorenstein and $K_{C}$ restricted to each $C_{i}$ is $K_{C_{i}}+2 x_{i}$ (see [Ca1, Proposition 1.18, (b), p. 64], or [Re, Theorem 3.7]), so that $\left|K_{C}\right|$ contracts the whole $(m-1)$-dimensional tangent space $T_{C, x}$ to a point.

A cluster $Z$ of degree 2 supported at $x$ corresponds to a point $Q \in$ $\mathbb{P}^{m-2}=\mathbb{P}\left(T_{C, x}\right)$. Since $Z$ is contracted by $K_{C}$ (together with the whole tangent space), by our usual argument, the group $\operatorname{Hom}\left(\mathcal{I}_{Z}, \mathcal{O}_{C}\right)$ is 2-dimensional and a general $s: \mathcal{I}_{Z} \rightarrow \mathcal{O}_{C}$ has image $\mathcal{I}_{Z^{\prime}}$ where $Z^{\prime}$ is a moving cluster of degree 2 at $x$, corresponding to a moving point $Q^{\prime} \in \mathbb{P}^{m-2}$. It is an amusing exercise to see that if $Q$ is linearly in general position with respect to the frame of reference $\left\{P_{1}, P_{2}, \ldots, P_{m}\right\}$ then $Q^{\prime}$ moves around the unique rational normal curve of degree $m-2$ passing through $\left\{P_{1}, P_{2}, \ldots, P_{m}, Q\right\}$. On the other hand, if $Z$ is in the tangent cone to $C$ (say, tangent to the branch $C_{1}$ ), then $\mathcal{I}_{Z}$ is not isomorphic to any other cluster of degree 2 , so that $\operatorname{Hom}\left(\mathcal{I}_{Z}, \mathcal{O}_{C}\right)=\operatorname{End}\left(\mathcal{I}_{Z}\right)$; this has 2 idempotents vanishing on $C_{1}$ and on $C_{2}+\cdots+C_{m}$. 
The following easy exercises may help to clarify things for the reader:

1. Let $x \in C$ be an ordinary triple point of a plane curve, say defined by an equation $f(u, v)=u^{3}+v^{3}+$ higher order terms; then for general $\lambda$, the ideals $\left(u+\lambda v, v^{2}\right)$ in $\mathcal{O}_{C, x}$ are all locally isomorphic. [Hint: Multiply by the rational function $(u+\mu v) /(u+\lambda v)$.]

2. If $C$ is the planar curve defined by $v w=v^{3}+w^{3}$ then $m_{x}=(v, w)$ is locally isomorphic to $\mathcal{I}_{Z}=\left(v, w^{2}\right)$ and to $\mathcal{I}_{Z^{\prime}}=\left(v^{2}, w\right)$.

3. If $C$ is the planar curve locally defined by $v^{2}=w^{3}$ then $m_{x}=(v, w)$ is locally isomorphic to $\mathcal{I}_{Z}=\left(v, w^{2}\right)$.

(Compare the proof of Proposition 6.9, Step 6.)

\section{LemMa 6.4. Case 2 is impossible.}

Proof. Since $x \in Z \cap Z^{\prime}$ and $\operatorname{Supp} Z \neq \operatorname{Supp} Z^{\prime}$, we can interchange $Z$ and $Z^{\prime}$ if necessary and assume that $Z^{\prime}=\{x, y\}$ with $x \neq y$. Consider the inclusion $s: \mathcal{I}_{Z} \hookrightarrow \mathcal{O}_{C}$ with image $s\left(\mathcal{I}_{Z}\right)=\mathcal{I}_{Z^{\prime}}=m_{x} m_{y}$ and the identity inclusion. One of these vanishes at $y$ and the other doesn't, so their restrictions to a component $\Gamma$ containing $y$ are linearly independent on $\Gamma$, and, as in Claim 3.7, for any general point $y^{\prime} \in \Gamma$, some linear combination $s^{\prime}=s+\lambda$ id defines an isomorphism $s^{\prime}: \mathcal{I}_{Z} \cong m_{x} m_{y^{\prime}}$. Reversing our usual argument shows that $x$ and $y^{\prime}$ are contracted to the same point by $\left|K_{C}\right|$ or $\left|2 K_{X}\right|$, so that the free linear system $\left|2 K_{X}\right|$ contracts $\Gamma$ to a point. This contradicts $K_{X}$ ample.

Q.E.D.

\section{Clusters on singular curves}

Our immediate aim is to exclude Case 1, but at the same time we introduce some ideas and notation used throughout the rest of this section. Choose a point $x \in Z$. Since $X$ has at worst hypersurface singularities and $C$ is a Cartier divisor in $X$, it is a local complete intersection, that is, locally defined by $F=G=0$. (Of course, $X$ may be nonsingular.) We think of $x \in Z \subset C \subset X \subset \mathbb{A}^{3}$ as local, and write $\mathcal{O}_{\mathbb{A}^{3}}, \mathcal{O}_{C}$, etc. for the local rings at $x$. We take local coordinates $u, v, w$ in $\mathbb{A}^{3}$ so that $Z$ is defined by $u=v=w=0$ in the reduced case, or $u=v=w^{2}=0$ otherwise. 
LEMMA 6.5.

(1) The quotient $\mathcal{I}_{\mathbb{A}^{3}, Z} / m_{\mathbb{A}^{3}, x} \mathcal{I}_{\mathbb{A}^{3}, Z}$ is a 3-dimensional vector space, and $Z \subset C$ is a Cartier divisor at $x$ if and only if $F, G$ map to linearly independent elements of it.

(2) Suppose that $p_{g} \geq 4$ and $Z$ is contracted by $\left|2 K_{X}\right|$. Then the curve $C \in\left|K_{X}-Z\right|$ can be chosen such that $Z$ is not a Cartier divisor. For this $C$, Case 1 is excluded.

Proof. (1) says that a minimal set of generators of the ideal $\mathcal{I}_{\mathbb{A}^{3}, Z}$ consists of 3 elements, which is obvious because $\mathcal{I}_{\mathbb{A}^{3}, Z}$ is locally generated at $x \in Z$ by the regular sequence $(u, v, w)$ or $\left(u, v, w^{2}\right)$. Now $Z$ is a Cartier divisor on $C$ if and only if $\mathcal{I}_{C, Z}$ is generated by 1 element, that is, $F$ and $G$ provide two of the minimal generators of $\mathcal{I}_{\mathbb{A}^{3}, Z}$. This proves (1).

For (2), suppose that $F=0$ is the local equation of $X \subset \mathbb{A}^{3}$. If $F \in m_{\mathbb{A}^{3}, x} \mathcal{I}_{\mathbb{A}^{3}, Z}$ then by (1), $Z$ is not a Cartier divisor on any curve $C \in\left|K_{X}-Z\right|$. Suppose then that $F \notin m_{\mathbb{A}^{3}, x} \mathcal{I}_{\mathbb{A}^{3}, Z}$, so that $F$ provides one of the minimal generators of $\mathcal{I}_{\mathbb{A}^{3}, Z}$. Then the ideal $\mathcal{I}_{X, Z}$ of $Z \subset X$ is generated by 2 elements, in other words, $\operatorname{dim}_{k} \mathcal{I}_{X, Z} / m_{X, x} \mathcal{I}_{X, Z}=2$. Therefore

$$
h^{0}\left(m_{x} \mathcal{I}_{Z} \mathcal{O}_{X}\left(K_{X}\right)\right) \geq h^{0}\left(\mathcal{I}_{Z} \mathcal{O}_{X}\left(K_{X}\right)\right)-2 \geq p_{g}-3 \geq 1
$$

(by remark (i) at the beginning of this section). Thus we can find a curve $C \in\left|K_{X}-Z\right|$ whose local equation at $x$ is $g \in m_{X, x} \mathcal{I}_{X, Z}$. Then $g$ has a local lift $G \in m_{\mathbb{A}^{3}, x} \mathcal{I}_{\mathbb{A}^{3}, Z}$, so that (1) applies to $C$.

Q.E.D.

Remark 6.6. The same argument can be expressed more geometrically. If $Z$ contains $x$ as a reduced point, that is, $\mathcal{I}_{\mathbb{A}^{3}, Z}=m_{x}$, then $x \in C$ is Cartier if and only if $C$ defined by $(F, G)$ is nonsingular at $x$, that is, $F, G$ map to linearly independent elements of $m_{x} / m_{x}^{2}$.

To interpret the nonreduced case $\mathcal{I}_{\mathbb{A}^{3}, Z}=\left(u, v, w^{2}\right)$, note that

$$
F \notin m_{\mathbb{A}^{3}, x} \mathcal{I}_{\mathbb{A}^{3}, Z} \Longleftrightarrow F=P u+Q v+R w^{2} \text { with one of } P, Q, R \notin m_{x} .
$$

In other words, the surface $Y$ locally defined by $F=0$ is either nonsingular at $x$, or has a double point with $Z$ not in the tangent cone. In the opposite case $F \in m_{\mathbb{A}^{3}, x} \mathcal{I}_{\mathbb{A}^{3}, Z}$, it is easy to see that $x \in C$ is either a complete intersection defined by two singular hypersurfaces, so has 3-dimensional tangent space $T_{C, x}$, or is a planar curve, which is either a double point with $Z$ in the tangent cone, or a point of multiplicity $\geq 3$. 


\section{The nilpotent-idempotent lemma}

Our proof of Theorem 1.4, (b) in Cases 3-4 is based on the following result. Note first that $\operatorname{Hom}\left(\mathcal{I}_{Z}, \mathcal{O}_{C}\right) \subset H^{0}\left(C \backslash \operatorname{Supp} Z, \mathcal{O}_{C}\right)$, and the latter is a ring. (We usually write $\mathcal{I}_{Z}$ for $\mathcal{I}_{C, Z}$ in what follows.) In other words, maps $\mathcal{I}_{Z} \rightarrow \mathcal{O}_{C}$ can be viewed as rational sections of $\mathcal{O}_{C}$ that are regular outside $\operatorname{Supp} Z$, so that it is meaningful to multiply them (the product is again a rational section of $\mathcal{O}_{C}$ that is regular away from $Z$ ).

Lemma 6.7. Assume that $K_{X}^{2} \geq 10$, and let $C \in\left|K_{X}-Z\right|$. Suppose that $s: \mathcal{I}_{Z} \rightarrow \mathcal{O}_{C}$ is a nonzero homomorphism which is either nilpotent with $s^{4}=0$, or a nontrivial idempotent with $s(1-s)=0$. Then the scheme theoretic support of $s$ (respectively, in the idempotent case, either $s$ or $1-s$ ) is a Francia curve $B$, and $\mathcal{I}_{Z} \mathcal{O}_{B}\left(2 K_{X}\right) \cong \omega_{B}$.

More generally, suppose that $s_{i}: \mathcal{I}_{Z} \rightarrow \mathcal{O}_{C}$ for $i=1, \ldots, 4$ are nonzero homomorphisms such that $s_{1} s_{2} s_{3} s_{4}=0$. Then one of the $s_{i}$ has scheme theoretic support a Francia curve $B_{i}$ with $\mathcal{I}_{Z} \mathcal{O}_{B_{i}}\left(2 K_{X}\right) \cong \omega_{B_{i}}$.

The final part is more general, because we allow some $s_{i}=\mathrm{id}$, or some of the $s_{i}$ to coincide. Notice that $\mathcal{O}_{C}$ has no sections supported at finitely many points, so we need only check the conditions $s^{4}=0$ etc. in each generic stalk of $\mathcal{O}_{C}$, that is, as rational functions on $C$.

Proof. If $s: \mathcal{I}_{Z} \mathcal{O}_{C}\left(K_{C}\right) \rightarrow \omega_{C}$ is not generically injective, the factorisation provided by automatic adjunction (Lemma 2.4) gives a subcurve $B \subset C$ satisfying $\mathcal{I}_{Z} \mathcal{O}_{B}\left(K_{C}\right) \cong \omega_{B}$; we are in the limiting case of numerically 2-connected. Write $C=A+B$ for the decomposition of Weil divisors, so that $A$ is the divisor of zeros of $s$. Passing to the minimal nonsingular model $S$ and taking the hat transform $\widehat{B}$ as in Lemma 4.2 and Claim 4.3 gives a decomposition $K_{S} \stackrel{\operatorname{lin}}{\sim} f^{*} C=A_{1}+\widehat{B}$ such that $A_{1} \widehat{B}=2$.

Therefore by the Hodge algebraic index theorem, $A_{1}^{2} \widehat{B}^{2} \leq\left(A_{1} \widehat{B}\right)^{2}=4$. If both $A_{1}^{2}, \widehat{B}^{2} \geq 1$, it follows that $K_{S}^{2} \leq 9$, a contradiction, so that either $A_{1}^{2} \leq 0$ or $\widehat{B}^{2} \leq 0$. Then (because $K_{S}=A_{1}+\widehat{B}$ and $A_{1} \widehat{B}=2$ ), either $K_{X} \bar{A}=K_{S} A_{1} \leq 2$ or $K_{X} B=K_{S} \widehat{B} \leq 2$. Suppose for the moment that $K_{S} \widehat{B} \leq 2$. Since $2 p_{a} \widehat{B}-2=\widehat{B}^{2}+K_{S} \widehat{\widehat{B}}$, it follows at once that we are in one of the two cases

$$
\widehat{B}^{2}=-1, K_{S} \widehat{B}=1, p_{a} \widehat{B}=1 \text { or } \widehat{B}^{2}=0, K_{S} \widehat{B}=2, p_{a} \widehat{B}=2 .
$$

But by Lemma 4.2 and Claim 4.3 we have $K_{S} \widehat{B}=K_{X} B$ and $p_{a} \widehat{B}=p_{a} B$, so that $B$ is the required Francia curve. 
It remains to get rid of the possibility that $K_{X} A=K_{S} A_{1} \leq 2$ in the different cases. If $s$ is a nontrivial idempotent, we can swap $A \leftrightarrow B$ by $s \leftrightarrow 1-s$ if necessary, so that $K_{X} B \leq 2$. In the nilpotent case, since $A$ equals the Weil divisor of zeros of $s$ and $s^{4}=0$, it follows that $C \leq 4 A$. Then $K_{X} A \leq 2$ would imply $K_{X}^{2} \leq 8$, a contradiction.

The last part is exactly the same: each $s_{i}$ (for $i=1,2,3,4$ ) is either injective or has scheme theoretic support a subcurve $B_{i} \subset C$ with $\mathcal{I}_{Z} \mathcal{O}_{B_{i}}\left(K_{C}\right) \cong \omega_{B_{i}}$, and divisor of zeros $A_{i}=C-B_{i}$. Since $\prod s_{i}=0$ it follows that $C \leq \sum A_{i}$. Now arguing as above gives that one of $K_{X} A_{i}$ or $K_{X} B_{i} \leq 2$; if the first alternative holds for all $i$ then $K_{X}^{2}=K_{X} C \leq$ $\sum K_{X} A_{i} \leq 8$, a contradiction. This proves the lemma. Q.E.D.

We apply Lemma 6.7 via a simple algebraic trick.

Corollary 6.8. If $A=\operatorname{End}_{\mathcal{O}_{C}}\left(\mathcal{I}_{C, Z}\right)$ is an Artinian algebra of length $\geq 2$ then it has a nontrivial idempotent or a nonzero nilpotent with $s^{2}=0$. More generally, if $\operatorname{Hom}\left(\mathcal{I}_{C, Z}, \mathcal{O}_{C}\right)$ is a 2-dimensional vector space contained in an Artinian algebra $A \subset H^{0}\left(C \backslash \operatorname{Supp} Z, \mathcal{O}_{C}\right)$ of dimension $\leq 4$ then there exist nonzero elements $s_{1}, \ldots, s_{4} \in \operatorname{Hom}\left(\mathcal{I}_{C, Z}, \mathcal{O}_{C}\right)$ with zero product. Under either assumption, Lemma 6.7 gives a Francia curve $B \subset C$ containing $Z$.

This completes the proof of Theorem 1.4, (b) in Case 3, since the case assumption is that $s: \mathcal{I}_{Z} \rightarrow \mathcal{I}_{Z} \subset \mathcal{O}_{C}$, so that $\operatorname{Hom}\left(\mathcal{I}_{Z}, \mathcal{O}_{C}\right)=\operatorname{End}\left(\mathcal{I}_{Z}\right)$ is a 2-dimensional Artinian algebra.

Proof. In the main case $\operatorname{dim} A=2$, this is completely trivial: if $k \subset A$ is the constant subfield, any $s \in A \backslash k$ satisfies a quadratic equation over $k$ of the form

$$
0=s^{2}+a s+b=(s-\alpha)(s-\beta) .
$$

If $\alpha=\beta$ then $s^{\prime}=s-\alpha$ is nilpotent with $s^{\prime 2}=0$; otherwise, $s^{\prime}=$ $(s-\alpha) /(\alpha-\beta)$ and $1-s^{\prime}=(s-\beta) /(\beta-\alpha)$ are orthogonal idempotents.

More generally, an Artinian algebra is a product $A=A_{1} \times \cdots \times A_{l}$ with local Artinian rings $\left(A_{i}, n_{i}\right)$ as factors; the maximal ideals of $A$ are codimension 1 vector subspaces $m_{i} \subset A_{i}$ given by $n_{1} \times A_{2} \times \cdots \times A_{l}$ (say). The projection to the factors (if $l \geq 2$ ) give nontrivial idempotents; if $l=1$ then $A$ is local, with nilpotent maximal ideal. This proves the first part.

We now prove the more general statement: a 2-dimensional vector subspace $V \subset A$ in an Artinian algebra has nonzero intersection with every maximal ideal, say $s_{i} \in V \cap m_{i}$. If the local factors $\left(A_{i}, n_{i}\right)$ have dimension 
$d_{i}$ then $n_{i}^{d_{i}}=0$, and the product $\prod s_{i}^{d_{i}}$ maps to zero in each factor, so is zero in $A$. Taking $\sum d_{i}=\operatorname{dim} A \leq 4$ gives the final part of the claim. Q.E.D.

\section{Proof in Case 4}

In the following proposition, $x \in C \subset \mathbb{A}^{3}$ is a local curve which is a local complete intersection at $x$. We choose local coordinates $u, v, w$ on $\mathbb{A}^{3}$ so that $\mathcal{I}_{\mathbb{A}^{3}, Z} \subset \mathcal{O}_{\mathbb{A}^{3}}$ is generated at $x$ by the regular sequence $u, v, w^{2}$. As before, we write $\mathcal{O}_{C}$ for the local ring $\mathcal{O}_{C, x}$ and $\mathcal{I}_{Z}=\mathcal{I}_{C, Z}$ for the $\mathcal{O}_{C}$ module obtained as the stalk at $x$ of the corresponding ideal sheaf. (Thus the statement of the proposition only concerns homomorphisms $s: \mathcal{I}_{Z} \rightarrow \mathcal{O}_{C}$ of modules over the local ring $\mathcal{O}_{C}$.)

Proposition 6.9. Let $Z \subset C$ be a cluster of degree 2 supported at $x$. We assume

(i) $Z$ is not a Cartier divisor on $C$;

(ii) there exists a homomorphism $s_{0}: \mathcal{I}_{Z} \rightarrow \mathcal{O}_{C}$ such that for general $\lambda \in k$, $s_{0}+\lambda$ id defines an isomorphism $\mathcal{I}_{Z} \cong \mathcal{I}_{Z_{\lambda}}$ with $Z_{\lambda}$ a cluster of degree 2 supported at $x$, and $Z_{0} \neq Z$.

Then any homomorphism $s: \mathcal{I}_{Z} \rightarrow \mathcal{O}_{C}$ takes $m_{C, x}^{2}$ to $m_{C, x}^{2}$, that is,

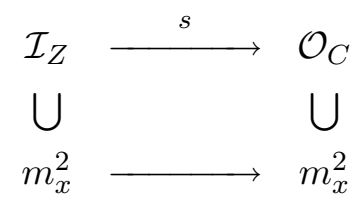

Proof of Theorem 1.4, (b) in Case 4. We apply the proposition to the global homomorphism $s: \mathcal{I}_{Z} \rightarrow \mathcal{O}_{C}$, using the assumption of Case 4 . We get

$$
\operatorname{Hom}\left(\mathcal{I}_{Z}, \mathcal{O}_{C}\right) \subset \operatorname{End}\left(m_{x}^{2}\right) \subset \operatorname{Hom}\left(m_{x}^{2}, \mathcal{O}_{C}\right) .
$$

Now Lemma 6.2 gives $\operatorname{dim} \operatorname{Hom}\left(\mathcal{I}_{Z}, \mathcal{O}_{C}\right)=2$ and $\operatorname{dim} \operatorname{Hom}\left(m_{x}^{2}, \mathcal{O}_{C}\right) \leq 4$; but $A=\operatorname{End}\left(m_{x}^{2}\right)$ is a subring of $H^{0}\left(C \backslash \operatorname{Supp} Z, \mathcal{O}_{C}\right)$, so that Corollary 6.8 gives the result.

Q.E.D.

Proof of Proposition 6.9, Step 1. If $s \in \operatorname{Hom}\left(\mathcal{I}_{Z}, \mathcal{O}_{C}\right)$ is any element then $s\left(\mathcal{I}_{Z}\right) \subset m_{x}$; for otherwise $s$ would be an isomorphism $\mathcal{I}_{Z} \cong \mathcal{O}_{C}$ near $x$, contradicting the assumption that $Z \subset C$ is not Cartier. 
Step 2. Note that $m_{x}^{2} \subset \mathcal{I}_{Z}$, so that we can restrict $s: \mathcal{I}_{Z} \rightarrow \mathcal{O}_{C}$ to $m_{x}^{2}$. Also, $m_{x} \mathcal{I}_{Z} \subset m_{x}^{2}$, and obviously $s\left(\mathcal{I}_{Z}\right) \subset m_{x}$ implies that $s\left(m_{x} \mathcal{I}_{Z}\right) \subset m_{x}^{2}$.

Step 3. It is enough to prove that $s\left(w^{2}\right) \in m_{x}^{2}$. Indeed,

$$
m_{x} \mathcal{I}_{Z}=(u, v, w) \cdot\left(u, v, w^{2}\right)=\left(u^{2}, u v, v^{2}, u w, v w, w^{3}\right),
$$

so that

$$
m_{x}^{2}=(u, v, w)^{2}=\left(u^{2}, u v, v^{2}, u w, v w, w^{2}\right)=m_{x} \mathcal{I}_{Z}+\mathcal{O}_{C} w^{2} \subset \mathcal{O}_{C} .
$$

Step 4. Since $C$ is a local complete intersection, $\mathcal{I}_{\mathbb{A}^{3}, C}=(F, G)$, where $F, G \in \mathcal{O}_{\mathbb{A}^{3}}$ is a regular sequence. Now $Z \subset C$ gives $F, G \in \mathcal{I}_{\mathbb{A}^{3}, Z}$, so that

$$
\begin{aligned}
& F=P u+Q v+R w^{2}, \\
& G=P^{\prime} u+Q^{\prime} v+R^{\prime} w^{2}, \quad \text { with } \quad P, Q, R, P^{\prime}, Q^{\prime}, R^{\prime} \in \mathcal{O}_{\mathbb{A}^{3}} .
\end{aligned}
$$

The set of local homomorphisms $\mathcal{I}_{Z} \rightarrow \mathcal{O}_{C}$ is a module over $\mathcal{O}_{C}$; this is the stalk at $x$ of the sheaf $\mathcal{H o m}$. For the moment, we take on trust the following general fact (see Appendix to $\S 6$ for a discussion and a detailed proof.)

Claim 6.10. The $\mathcal{O}_{C}$ module $\mathcal{H o m}_{\mathcal{O}_{C}}\left(\mathcal{I}_{Z}, \mathcal{O}_{C}\right)$ is generated by two elements, the identity inclusion id: $\mathcal{I}_{C, Z} \hookrightarrow \mathcal{O}_{C}$ and the map $t: \mathcal{I}_{C, Z} \rightarrow \mathcal{O}_{C}$ determined by the minors of the $2 \times 3$ matrix of coefficients of $F, G$ :

$$
t(u)=Q R^{\prime}-R Q^{\prime}, \quad t(v)=-P R^{\prime}+R P^{\prime}, \quad t\left(w^{2}\right)=P Q^{\prime}-Q P^{\prime} .
$$

Step 5. According to Steps 3-4, to prove Proposition 6.9, we need only prove that $P Q^{\prime}-Q P^{\prime} \in m_{\mathbb{A}^{3}, x}^{2}$. We are home if all four of $P, Q, P^{\prime}, Q^{\prime} \in m_{x}$. Thus in what follows, we assume (say) that $P^{\prime} \notin m_{x}$. Then $P^{\prime}$ is a unit, and $G=0$ defines a nonsingular surface $Y$ containing $C$. Dividing by $P^{\prime}$, we can rewrite $G$ in the form $u=-\left(Q^{\prime} / P^{\prime}\right) v-\left(R^{\prime} / P^{\prime}\right) w^{2}$. Then subtracting a multiple of this relation from $F$ gives $f=q v+r w^{2}$ as the local equation of $C \subset Y$ (where $q=Q-P Q^{\prime} / P^{\prime}$ and $r=R-P R^{\prime} / P^{\prime}$ ).

Therefore it only remains to prove that if $C$ is the planar curve defined by $f=q v+r w^{2}$, the two assumptions of Proposition 6.9 imply that $q \in$ $m_{Y, x}^{2}$. As in Lemma 6.5, assumption (i) implies that $q, r \in m_{Y, x}$, so that $q \in m_{Y, x}^{2}$ is equivalent to saying that $x \in C \subset Y$ has multiplicity $\geq 3$. 
Step 6. Consider the linear terms of the given isomorphism $s_{0}: \mathcal{I}_{Z} \rightarrow$ $\mathcal{I}_{Z_{0}}$ :

$$
s_{0}(v)=a v+b w \bmod m_{Y, x}^{2}, \quad s_{0}\left(w^{2}\right)=c v+d w \bmod m_{Y, x}^{2} .
$$

Because $Z_{0} \neq Z$, it follows that $(b, d) \neq(0,0)$. However, if $b=0$ and $d \neq 0$, then for general $\lambda$, the two generators of $\mathcal{I}_{Z_{\lambda}}=\left(s_{0}(v)+\lambda v, s\left(w^{2}\right)+\lambda w^{2}\right)$ would have linearly independent linear terms, so that $\mathcal{I}_{Z_{\lambda}}=m_{C, x}$. This contradicts assumption (ii). Therefore $b \neq 0$, and $\mathcal{I}_{Z_{\lambda}}$ has a generator with the variable linear term $(a+\lambda) v+b w$. It follows that $Z_{\lambda}$ runs linearly around the tangent space to $x$ in $C$.

Now we claim that $x \in C \subset Y$ is a planar curve singularity of multiplicity $\geq 3$. Indeed, the isomorphism $\mathcal{I}_{Z} \cong \mathcal{I}_{Z_{\lambda}}$ implies that $Z_{\lambda} \subset C$ cannot be a Cartier divisor; but if $x \in C \subset Y$ were a double point, this would restrict $Z_{\lambda}$ to be in the tangent cone, contradicting what we have just proved. This completes the proof of Proposition 6.9.

Q.E.D.

\section{Appendix: Proof of Claim 6.10}

We start by slightly generalising the set-up: let $\mathcal{O}_{\mathbb{A}}$ be a local ring, assumed to be regular (for simplicity only), and $x, y, z$ a regular sequence generating a codimension 3 complete intersection ideal $\mathcal{I}_{Z}=(x, y, z)$. Consider a regular sequence $F, G \in \mathcal{I}_{Z}$. Note that

$$
F=P x+Q y+R z \text { and } G=P^{\prime} x+Q^{\prime} y+R^{\prime} z
$$

for some $P, \ldots, R^{\prime} \in \mathcal{O}_{\mathbb{A}}$. Write $\mathcal{O}_{C}=\mathcal{O}_{\mathbb{A}} /(F, G)$ and $\mathcal{I}_{C, Z}=\mathcal{I}_{Z} \mathcal{O}_{C}=$ $(x, y, z) \subset \mathcal{O}_{C}$. (In the application, $Z \subset \mathbb{A}=\mathbb{A}^{3}$ was a nonreduced cluster defined by $(x, y, z)=\left(u, v, w^{2}\right)$ and $C \subset \mathbb{A}^{3}$ a complete intersection curve through $Z$.)

LEMMA 6.11.

(1) A presentation of $\mathcal{I}_{C, Z}$ over $\mathcal{O}_{C}$ is given by

$$
\mathcal{O}_{C}^{\oplus 5} \stackrel{M}{\longrightarrow} \mathcal{O}_{C}^{\oplus 3} \stackrel{\left(\begin{array}{l}
x \\
y \\
z
\end{array}\right)}{\longrightarrow} \mathcal{I}_{C, Z} \longrightarrow 0 \text {, where } M=\left(\begin{array}{ccc}
P & Q & R \\
P^{\prime} & Q^{\prime} & R^{\prime} \\
0 & z & -y \\
-z & 0 & x \\
y & -x & 0
\end{array}\right) .
$$


(2) $\operatorname{Hom}\left(\mathcal{I}_{C, Z}, \mathcal{O}_{C}\right)$ is generated over $\mathcal{O}_{C}$ by the two elements id and $t$, where

$$
t:\left(\begin{array}{c}
x \\
y \\
z
\end{array}\right) \longmapsto\left(\begin{array}{r}
Q R^{\prime}-R Q^{\prime} \\
-P R^{\prime}+R P^{\prime} \\
P Q^{\prime}-Q P^{\prime}
\end{array}\right) .
$$

Proof. (1) An almost obvious calculation: because $\mathcal{I}_{C, Z}=(x, y, z)$, there is a surjective map $\varphi: \mathcal{O}_{C}^{\oplus 3} \rightarrow \mathcal{I}_{C, Z}$, such that $\left(h_{1}, h_{2}, h_{3}\right) \in \operatorname{ker} \varphi$ if and only if $h_{1} x+h_{2} y+h_{3} z=0 \in \mathcal{O}_{C}$. Write $H_{1}, H_{2}, H_{3} \in \mathcal{O}_{\mathbb{A}}$ for lifts of the $h_{i}$. Then $H_{1} x+H_{2} y+H_{3} z \in \mathcal{I}_{\mathbb{A}^{3}, C}=(F, G)$. Subtracting off multiples of $F$ and $G$ means exactly subtracting multiples of the first two rows of $M$ from $\left(H_{1}, H_{2}, H_{3}\right)$, to give identities $H_{1}^{\prime} x+H_{2}^{\prime} y+H_{3}^{\prime} z=0 \in \mathcal{O}_{\mathbb{A}}$. Now $x, y, z \in \mathcal{O}_{C}$ is a regular sequence, so it follows that $\left(H_{1}^{\prime}, H_{2}^{\prime}, H_{3}^{\prime}\right)$ is in the image of the Koszul matrix given by the bottom 3 rows of $M$. This proves (1).

(2) A homomorphism $s: \mathcal{I}_{C, Z} \rightarrow \mathcal{O}_{C}$ is determined by $(x, y, z) \mapsto(a, b, c)$ where $a, b, c \in \mathcal{O}_{C}$ satisfy $M(a, b, c)^{\operatorname{tr}}=0$ (we write $(a, b, c)^{\operatorname{tr}}$ for the column vector). It is easy to check that $(7)$ gives a map $t$ in this way.

The condition $M(a, b, c)^{\operatorname{tr}}=0$ consists of 5 equalities in $\mathcal{O}_{C}=$ $\mathcal{O}_{\mathbb{A}} /(F, G)$. We choose lifts $A, B, C$ to $\mathcal{O}_{\mathbb{A}}$, and write out the last 3 of these as identities in $\mathcal{O}_{\mathbb{A}}$ :

$$
\begin{aligned}
-z B+y C & =\alpha F-\alpha^{\prime} G \\
z A-x C & =\beta F-\beta^{\prime} G \quad \text { for some } \alpha, \ldots, \gamma^{\prime} \in \mathcal{O}_{\mathbb{A}} . \\
-y A+x B \quad & =\gamma F-\gamma^{\prime} G
\end{aligned}
$$

Taking $x$ times the first plus $y$ times the second plus $z$ times the third, the left-hand sides cancel, giving the identity

$$
(\alpha x+\beta y+\gamma z) F=\left(\alpha^{\prime} x+\beta^{\prime} y+\gamma^{\prime} z\right) G \in \mathcal{O}_{\mathbb{A}} .
$$

Now since $F, G$ is a regular sequence in $\mathcal{O}_{\mathbb{A}}$, this implies that

$$
\begin{aligned}
\alpha x+\beta y+\gamma z & =D G=D\left(P^{\prime} x+Q^{\prime} y+R^{\prime} z\right) \\
\alpha^{\prime} x+\beta^{\prime} y+\gamma^{\prime} z & =D F=D(P x+Q y+R z)
\end{aligned}
$$

for some $D \in \mathcal{O}_{\mathbb{A}}$.

Now subtracting $D$ times the given generator $t$ changes

$$
\left(\begin{array}{c}
A \\
B \\
C
\end{array}\right) \longmapsto\left(\begin{array}{c}
A \\
B \\
C
\end{array}\right)-\left(\begin{array}{c}
Q R^{\prime}-Q^{\prime} R \\
-P R^{\prime}+P^{\prime} R \\
P Q^{\prime}-P^{\prime} Q
\end{array}\right) D
$$


and has the following effect on the quantities $\alpha, \ldots, \gamma^{\prime}$ introduced in (8):

$$
\begin{aligned}
(\alpha, \beta, \gamma) & \longmapsto\left(\alpha+D P^{\prime}, \beta+D Q^{\prime}, \gamma+D R^{\prime}\right) \\
\left(\alpha^{\prime}, \beta^{\prime}, \gamma^{\prime}\right) & \longmapsto\left(\alpha^{\prime}+D P, \beta^{\prime}+D Q, \gamma^{\prime}+D R\right)
\end{aligned}
$$

To see this, note that the first equation of (8) is

$$
-z B+y C=\alpha F-\alpha^{\prime} G=\alpha(P x+Q y+R z)-\alpha^{\prime}\left(P^{\prime} x+Q^{\prime} y+R^{\prime} z\right),
$$

so that the effect of the two substitutions $\alpha \mapsto \alpha+D P^{\prime}$ and $\alpha^{\prime} \mapsto \alpha^{\prime}+D P$ on the right exactly cancels out $B \mapsto B+D\left(P R^{\prime}-P^{\prime} R\right)$ and $C \mapsto C-$ $D\left(P Q^{\prime}-P^{\prime} Q\right)$ on the left. The upshot is that we can assume $D=0$ in (9).

But then since $(x, y, z)$ is a regular sequence, $(9)$ with $D=0$ gives

$$
\begin{aligned}
& \alpha=\quad l y-m z \quad \alpha^{\prime}=\quad l^{\prime} y-m^{\prime} z \\
& \beta=-l x \quad+n z \quad \text { and } \quad \beta^{\prime}=-l^{\prime} x \quad+n^{\prime} z \\
& \gamma=m x-n y \quad \gamma^{\prime}=m^{\prime} x-n^{\prime} y
\end{aligned}
$$

for some $l, \ldots, n^{\prime} \in \mathcal{O}_{\mathbb{A}}$. Finally (8) can now be rearranged as

$$
\begin{aligned}
& \left(C-l F+l^{\prime} G\right) y=\left(B-m G+m^{\prime} G\right) z \\
& A-n F+n^{\prime} G=E x \\
& \left(C-l F+l^{\prime} G\right) x=\left(A-n F+n^{\prime} G\right) z \quad \text { therefore } B-m F+m^{\prime} G=E y \\
& \left(B-m F+m^{\prime} G\right) x=\left(A-n F+n^{\prime} G\right) y \quad C-l F+l^{\prime} G=E z
\end{aligned}
$$

for some $E \in \mathcal{O}_{\mathbb{A}}$. This means that the map $s$ given by $(a, b, c)$ is a linear combination of $t$ and the identity, as required.

Q.E.D.

A less pedestrian method of arguing is to say that all three of $\mathcal{O}_{\mathbb{A}}, \mathcal{O}_{C}$ and $\mathcal{O}_{Z}$ are Gorenstein, so that adjunction gives

$$
0 \longrightarrow \omega_{C} \longrightarrow \mathcal{H o m}\left(\mathcal{I}_{Z}, \omega_{C}\right) \longrightarrow \omega_{Z}=\operatorname{Ext}^{1}\left(\mathcal{O}_{Z}, \omega_{C}\right) \longrightarrow 0
$$

The two generators id and $t$ correspond naturally to the generators of $\omega_{C}$ and $\omega_{Z}$.

\section{REFERENCES}

[Ar1] M. Artin, Some numerical criteria for contractibility of curves on algebraic surfaces, Amer. J. Math., 84 (1962), 485-496.

[Ar2] On isolated rational singularities of surfaces, Amer. J. Math., 88 (1966), 129-136. 
[B-P-V] W. Barth, C. Peters and A. Van de Ven, Compact complex surfaces, Springer, 1984.

[Ba1] I. Bauer, Geometry of algebraic surfaces admitting an inner projection, Preprint Pisa no. 1.72, 708 (1992).

[Ba2] Embeddings of curves, Manuscr. Math., 87 (1995), 27-34.

[Ba3] - Inner projections of algebraic surfaces: a finiteness result., J. reine angew. Math., 460 (1995), 1-13.

[Ba4] The classification of surfaces in $\mathbb{P}^{5}$ having few trisecants, Rend. del Sem. Mat. di Torino., 56/1 (1998).

[Bo] E. Bombieri, Canonical models of surfaces of general type, Publ. Math. IHES, 42 (1973), 171-219.

[B-M] E. Bombieri and D. Mumford, Enriques' classification of surfaces in char $p$ II, in 'Complex Analysis and Algebraic Geometry', collected papers dedicated to K. Kodaira, Iwanami Shoten, Tokyo (1977), pp. 23-42.

[Ca1] F. Catanese, Pluricanonical Gorenstein curves, in 'Enumerative Geometry and Classical Algebraic Geometry', Nice, Prog. in Math. 24, Birkhäuser (1981), pp. 51-95.

[Ca2] Footnotes to a theorem of Reider, in 'Algebraic Geometry', Proceedings of the L'Aquila conference 1988. (A. J. Sommese, A. Biancofore, E. L. Livorni, eds.), Springer LNM 1417 (1990), pp. 67-74.

[C-F] F. Catanese and M. Franciosi, Divisors of small genus on algebraic surfaces and projective embeddings, Proceedings of the conference "Hirzebruch 65", Tel Aviv 1993, Contemp. Math., A.M.S. (1994), subseries 'Israel Mathematical Conference Proceedings' Vol. 9 (1996), 109-140.

[C-H] F. Catanese and K. Hulek, Rational surfaces in $\mathbb{P}^{4}$ containing a plane curves, Ann. Mat. Pura Appl. (4), 172 (1997), 229-256.

[C-C-M] F. Catanese, C. Ciliberto and M. Mendes Lopes, On the classification of irregular surfaces of general type with nonbirational bicanonical map, Trans. Amer. Math. Soc., 350 (1998), 275-308.

[C-F-M] C. Ciliberto, P. Francia and M. Mendes Lopes, Remarks on the bicanonical map for surfaces of general type, Math. Z., 224 (1997), 137-166.

[Ek] T. Ekedahl, Canonical models of surfaces of general type in positive characteristic, Publ. Math. IHES, 67 (1988), 97-144.

[F] M. Franciosi, On $k$-spanned surfaces of sectional genus 8, Publ. Dip. Mat., Univ. di Pisa 1.114, 846, 1995.

[Fr1] P. Francia, The bicanonical map for surfaces of general type, unpublished manuscript, c. 1982-1987.

[Fr2] On the base points of the bicanonical system, in 'Problems in the theory of surfaces and their classification' (Cortona, Oct. 1988) (F. Catanese and others, eds.), Symp. Math. XXXII, Acad. Press INDAM, Rome (1991), pp. 141-150.

[Gr-Ha] A. Grothendieck (notes by R. Hartshorne), Local cohomology, Springer, LNM 41, 1967.

[Ha] R. Hartshorne, Algebraic Geometry, Springer, 1977. 
[Mu] D. Mumford, The canonical ring of an algebraic surface, Ann. of Math., 76 (1962), 612-615.

[ML] M. Mendes Lopes, Adjoint systems on surfaces, Boll. Un. Mat. Ital. A (7), 10 (1996), 169-179.

[Ra] K. Ranestad, Surfaces of degree 10 in the projective fourspace, in 'Problems in the theory of surfaces and their classification' (Cortona, Oct. 1988) (F. Catanese and others, eds.), Symp. Math. XXXII, Acad. Press INDAM, Rome (1991), pp. 271-307.

[Re] M. Reid, Nonnormal del Pezzo surfaces, Publications of RIMS 30:5 (1995), 695-727.

[Rei] I. Reider, Vector bundles of rank 2 and linear systems on algebraic surfaces, Ann. of Math., 127 (1988), 309-316.

[Se1] J.-P. Serre, Faisceaux algébriques cohérents, Ann. of Math., 61 (1955), 197-278.

[Se2] Courbes algébriques et corps de classes, Hermann, Paris, 1959 (English translation, Springer, 1990).

[S-B] N. I. Shepherd-Barron, Unstable vector bundlesd and linear systems on surfaces in characteristic p, Invent. Math., 106 (1991), 243-262.

Fabrizio Catanese

Mathematisches Institut der Universität

Bunsenstrasse 3-5

D-37073 Göttingen

Germany

catanese@cfgauss.uni-math.gwdg.de

Marco Franciosi

Scuola Normale Superiore

piazza dei Cavalieri 7

I-56126 Pisa

Italy

francios@cibs.sns.it, francios@gauss.dm.unipi.it

Klaus Hulek

Institut für Mathematik, Univ. Hannover

Postfach 6009

D-30060 Hannover

Germany

hulek@math .uni-hannover.de

Miles Reid

Math Inst., Univ. of Warwick

Coventry $C V 47 A L$

England

Miles@Maths. Warwick.Ac.UK 